Claremont Colleges

Scholarship@ Claremont

All HMC Faculty Publications and Research

HMC Faculty Scholarship

$1-1-2004$

\title{
Blowup and Dissipation in a Critical-Case Unstable Thin Film Equation
}

Thomas P. Witelski

Duke University

Andrew J. Bernoff

Harvey Mudd College

Andrea L. Bertozzi

University of California - Los Angeles

\section{Recommended Citation}

T. P. WITELSKI, A. J. BERNOFF and A. L. BERTOZZI (2004). Blowup and dissipation in a critical-case unstable thin film equation. European Journal of Applied Mathematics, , pp 223-256. doi:10.1017/S0956792504005418.

This Article is brought to you for free and open access by the HMC Faculty Scholarship at Scholarship @ Claremont. It has been accepted for inclusion in All HMC Faculty Publications and Research by an authorized administrator of Scholarship @ Claremont. For more information, please contact scholarship@cuc.claremont.edu. 


\section{European Journal of Applied Mathematics}

http://journals.cambridge.org/EJM

Additional services for European Journal of Applied

Mathematics:

Email alerts: $\underline{\text { Click here }}$

Subscriptions: Click here

Commercial reprints: $\underline{\text { Click here }}$

Terms of use : $\underline{\text { Click here }}$

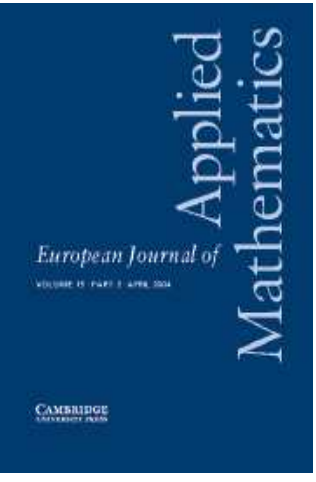

\section{Blowup and dissipation in a critical-case unstable thin film equation}

T. P. WITELSKI, A. J. BERNOFF and A. L. BERTOZZI

European Journal of Applied Mathematics / Volume 15 / Issue 02 / April 2004, pp 223 - 256

DOI: 10.1017/S0956792504005418, Published online: 07 June 2004

Link to this article: http://journals.cambridge.org/abstract_S0956792504005418

How to cite this article:

T. P. WITELSKI, A. J. BERNOFF and A. L. BERTOZZI (2004). Blowup and dissipation in a criticalcase unstable thin film equation. European Journal of Applied Mathematics, 15, pp 223-256 doi:10.1017/S0956792504005418

Request Permissions : $\underline{\text { Click here }}$ 


\title{
Blowup and dissipation in a critical-case unstable thin film equation
}

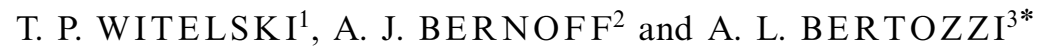 \\ ${ }^{1}$ Department of Mathematics and Center for Nonlinear and Complex Systems, Duke University, \\ Durham, NC 27708-0320, USA \\ email: witelski@math.duke.edu \\ ${ }^{2}$ Department of Mathematics, Harvey Mudd College, Claremont, CA 91711, USA \\ email: ajb@hmc.edu \\ ${ }^{3}$ Departments of Mathematics and Physics, Duke University, Durham, NC 27708-0320, USA \\ email: bertozzi@math.duke.edu
}

(Received 4 April 2003; revised 1 December 2003)

\begin{abstract}
We study the dynamics of dissipation and blow-up in a critical-case unstable thin film equation. The governing equation is a nonlinear fourth-order degenerate parabolic PDE derived from a generalized model for lubrication flows of thin viscous fluid layers on solid surfaces. There is a critical mass for blow-up and a rich set of dynamics including families of similarity solutions for finite-time blow-up and infinite-time spreading. The structure and stability of the steady-states and the compactly-supported similarity solutions is studied.
\end{abstract}

\section{Introduction}

This paper studies the dynamics of the fourth-order nonlinear parabolic partial differential equation

$$
\frac{\partial h}{\partial t}=-\frac{\partial}{\partial x}\left(h^{3} \frac{\partial h}{\partial x}\right)-\frac{\partial}{\partial x}\left(h \frac{\partial^{3} h}{\partial x^{3}}\right)
$$

for non-negative finite-mass initial data on a periodic domain, $-1 \leqslant x \leqslant 1$. We show that this problem has a rich structure including equilibrium solutions and continuous families of similarity solutions for both finite-time blow-up and self-similar spreading. There is a vast body of literature on similarity solutions and the formation of singularities in partial differential equations - for example see the references in $[2,3,13,30,63]$. Classic studies considered the dynamics of blow-up resulting from interactions between nonlinear terms and second-order spatial operators, as in the nonlinear Schrodinger equation $[21,28,46,48,55,60]$ and in semilinear heat equations $[2,20,29,30,31,32,33,34,47,57]$. The blow-up dynamics in (1.1) are governed by the the interaction between nonlinear second- and fourth-order terms, and as such it represents a higher-order analogue of these second-order model problems. The dynamics of (1.1) share many common features with the previous models, such as the existence of multi-bump similarity solutions $[21,22,55,56]$,

* Current address: Mathematics Department, UCLA, Box 951555, Los Angeles, CA 90095-1555, USA. Email: bertozzi@math.ucla.edu 
but the solutions of this nonlinear degenerate problem are weak compactly-supported 'droplet' solutions.

Equation (1.1) is a special case of the longwave-unstable generalized thin film equation,

$$
h_{t}=-\left(h^{m} h_{x}\right)_{x}-\left(h^{n} h_{x x x}\right)_{x}
$$

where $h(x, t)$ gives the height of the evolving free-surface. The exponents $m, n$ correspond to the powers in the destabilizing second-order and the stabilizing fourth-order diffusive terms, respectively. This class of model equation occurs in connection with many physical systems involving fluid interfaces $[50,53]$; when $n=1$ and $m=1$ it describes a thin jet in a Hele-Shaw cell $[1,13,23,25,36]$. When $n=3$ and $m=-1$ it describes van der Waals driven rupture of thin films [62, 65, 66, 67], and for $n=m=3$ it describes fluid droplets hanging from a ceiling [26]. For $n=0$ and $m=1$, equation (1.2) is a modified Kuramoto-Sivashinsky equation which describes solidification of a hyper-cooled melt $[8,17]$. Over the past 15 years, these models have also been the focus of rigorous and extensive mathematical analysis $[4,5,12,15,18,42,43,44,45,54]$.

In this paper, we focus on equation (1.2) with $n=1$ and $m=3$ because of its special role as a critical case between problems where solutions blow-up (see Figure 1) and problems whose solutions are bounded for all times. Questions on blow-up in (1.2) were raised by Hocherman \& Rosenau [39]. They proposed the existence of critical exponents that would separate those equations that have blow-up, $h \rightarrow \infty$, from those that do not. That is, if the strength of the destabilizing term is sufficient to overcome the regularizing influence of the fourth-order term, then blow-up can take place. The rigorous analysis of the blow-up problem was recently studied in two papers by Bertozzi \& Pugh [16, 17]. They proved [16] that blow-up is impossible for $m<n+2$, disproving the original conjecture of [39] that $m=n$ is critical. In the second paper [17], the special case $n=1$ is studied on the line for compactly supported initial data. They prove the existence of a solution that blows up in finite time for $m \geqslant 3=n+2$. Consequently, for $n=1, m=n+2=3$ is the critical exponent separating equations with possible finite-time blow-up from problems where the solutions are always bounded. As a critical case equation, (1.1) has many interesting characteristics that we will explore: a finite critical mass for blow-up, two classes of continuous families of self-similar solutions with compact support, and delicate interactions that can occur between pinch-off and blow-up. Our results build on a single framework that will serve to unify the dynamics of (1.1) in three regimes: ( $i$ ) near equilibrium, (ii) approaching finitetime blow-up, and (iii) infinite-time diffusive spreading. We will explore the connections between these different classes of solutions.

For much of our work, it is convenient to write (1.1) in a slightly different form, as

$$
\frac{\partial h}{\partial t}+\frac{\partial}{\partial x}\left(h \frac{\partial p}{\partial x}\right)=0, \quad p \equiv \frac{1}{3} h^{3}+\frac{\partial^{2} h}{\partial x^{2}},
$$

where $p$ defines a pressure function. This form stems from the interpretation of (1.1) as a generalization of the Reynolds lubrication equation for thin films of viscous fluids [53]. In this context, the terms in the pressure describe a body force on a thin fluid layer due to the cube of the thickness of the layer, and a surface tension contribution given by the linearized curvature of the free-surface of the layer, respectively. The pressure is a 


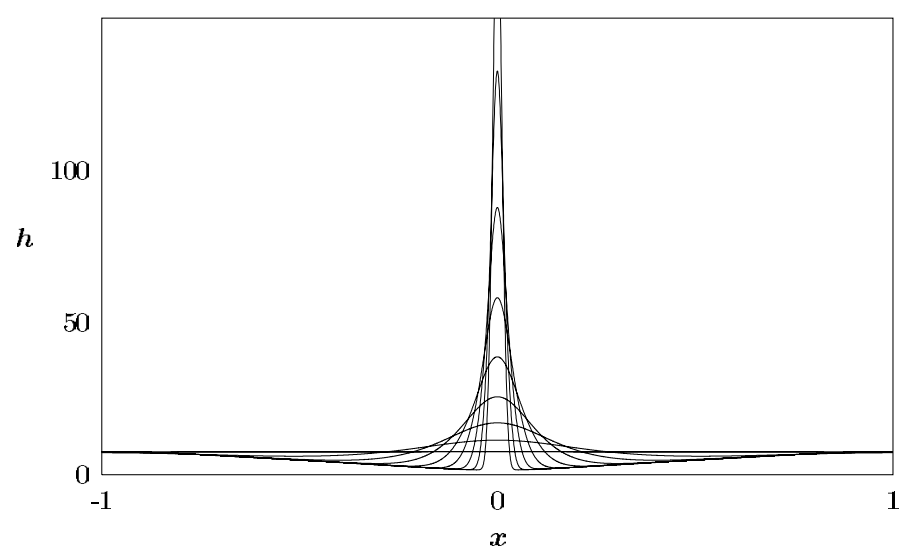

FIGURE 1. Finite-time blow-up for a solution of PDE (1.1) in a periodic domain.

key part of the analysis of the similarity solutions. We study initial value problems for (1.1) on the interval $-1 \leqslant x \leqslant 1$ with periodic boundary conditions, $h(x+1)=h(x-1)$, and non-negative initial data. Results from this problem can be related to the Neumann boundary value problem and the short-time dynamics of the Cauchy initial problem with compact initial data, under appropriate conditions [42, 43].

The total mass of the solution is given by

$$
M=\int_{-1}^{1} h d x
$$

the mass is conserved for all times. We shall use the mass as a control parameter to distinguish classes of initial data that will lead to different dynamics. Another fundamental global property of solutions of (1.1) is the monotone dissipation of the energy functional,

$$
\mathscr{E}=\int_{-1}^{1} \frac{1}{2} h_{x}^{2}-\frac{1}{12} h^{4} d x
$$

at the rate

$$
\frac{d \mathscr{E}}{d t}=-\int_{-1}^{1} h\left(\partial_{x} p\right)^{2} d x \leqslant 0 .
$$

It is possible to make use of the energy to describe: (i) the stability and dynamics of the solution [44]; and (ii) the evolution as a gradient flow in an appropriate weighted $H^{-1}$ norm [9].

The remainder of this article is as follows. In $\S 2$, we review results on blow-up of solutions with negative energy (1.5). We identify a critical mass $M_{c} \equiv 2 \pi \sqrt{2 / 3}$ below, which the solutions to (1.1) remain bounded for all time for both the periodic and Cauchy problems. In $\S 3$, we use dimensional analysis to obtain the scalings for the first-type similarity solutions of (1.1). The same set of similarity variables provide a framework for studying both classes of self-similar solutions: finite-time blow-up and infinite-time spreading. In $\S 4$, we examine the structure of these two classes of similarity solutions and the steady states of (1.1). These solutions all exist as equilibria of an equation we 
call the similarity PDE, which is a generalization of (1.3). The connections between these states are explored within this framework, somewhat analogously to the study of blow-up solutions and steady states [19]. In $\S 5$, the linear stability of these equilibria is analyzed. For the two classes of similarity solutions, the influence of the symmetries of the PDE must be considered in studying the spectrum $[9,63,66]$. Finally, in $\S 6$, further issues, problems, and open questions for the nonlinear dynamics of (1.1) are addressed using numerical simulations.

\section{Conditions on finite-time blow-up}

Perhaps the most dramatic behavior exhibited by solutions of (1.1) is that of finite-time blow-up, see Figure 1. The occurrence of blow-up can be noted from an argument based on the evolution of the second moment of the solution, as suggested by Bernoff [17],

$$
\frac{d}{d t}\left(\int x^{2} h d x\right)=-\frac{1}{2} \int h^{4} d x+3 \int h_{x}^{2} d x=6 \mathscr{E} .
$$

Since the energy (1.5) is monotone decreasing, (1.6), we have a bound on the evolution of the second moment in terms of the initial energy, $\mathscr{E}_{0}$,

$$
\frac{d}{d t}\left(\int x^{2} h d x\right) \leqslant 6 \mathscr{E}_{0} .
$$

If the initial energy is negative, then the second moment will become negative at a finite time. However, this is impossible since the solution is non-negative everywhere, $h(x, t) \geqslant 0$. The resolution to this apparent conflict is that (2.2) only applies while the solution $h(x, t)$ exists; if $h$ blows up at a finite time (before the second moment becomes negative) and ceases to exist thereafter, then there is no conflict. In fact, (2.2) yields an a priori upper bound on the critical time when blow-up will occur in terms of the initial data $h_{0}(x)$,

$$
t_{c} \leqslant \frac{1}{6\left|\mathscr{E}_{0}\right|} \int x^{2} h_{0} d x
$$

This formal argument applies to the Cauchy initial value problem with smooth initial data.

Rigorous results about blow-up for the Cauchy problem with compactly-supported initial data were obtained by Bertozzi \& Pugh [17] for (1.2) with $n=1$ and $m \geqslant 3$. There it was shown that blow-up can not occur for the periodic problem if the mass is sufficiently small. Indeed, Bertozzi and Pugh used dissipation of energy to observe that in the critical case, $m=n+2$ for all $n>0$, solutions are bounded for all time if their initial mass is sufficiently small [16, remark after Lemma 3.3]. Here we obtain a lower bound for the critical mass necessary for blow-up, $M_{c}$, for both the periodic problem and the Cauchy problems. In particular we can rigorously derive an a priori pointwise upper bound for the solution whenever the total mass is less than this $M_{c}$.

Theorem For solutions of the PDE (1.1) in either the periodic domain or $\mathbb{R}$, the boundedness of the energy functional (1.5) implies an a priori pointwise upper bound on the solution whenever the total mass $M=\int h d x$ is less than $M_{c} \equiv 2 \pi \sqrt{2 / 3}$. 
Proof The a priori bound derived in Bertozzi \& Pugh [17] relies on a classical GagliardoNirenberg estimate [27], which states that there exists $C$ such that

$$
\int h^{4} d x \leqslant C\left(\int|h| d x\right)^{2} \int h_{x}^{2} d x
$$

for all $h(x)$ in $H^{1}(\mathbb{R}) \cap L^{4}(\mathbb{R}) \cap L^{1}(\mathbb{R})$. Using this estimate for $h(x, t)$ in (1.5) yields

$$
\mathscr{E} \geqslant \frac{1}{2} \int h_{x}^{2} d x-\frac{C}{12}\left(\int h d x\right)^{2} \int h_{x}^{2} d x=\frac{6-C M^{2}}{12} \int h_{x}^{2} d x
$$

where $M$ is the mass of the solution, (1.4). Therefore, if $C M^{2}<6$ then $\mathscr{E}(t) \geqslant 0$, and the $H^{1}$ norm and thus the maximum of the solution is strictly bounded a priori. Note also that using this estimate we can show that if the integral of $h$ is less than $M_{c}$, then the energy is positive.

For the problem on $\mathbb{R}$, we can obtain a sharp value for $M_{c}$ by applying the following sharp inequality derived by Sz.-Nagy [49, 59].

Theorem (Sz.-Nagy [59]) Let $f^{\prime} \in L^{p}(\mathbb{R}), p>1$, and $f \in L^{1}(\mathbb{R})$. Then $f$ is in $L^{q}(\mathbb{R})$ for all $q>1$ and we have the estimate

$$
\left(\int_{-\infty}^{\infty}|f(x)|^{q} d x\right)^{1 / q} \leqslant K(q, r)\left(\int_{-\infty}^{\infty}|f(x)| d x\right)^{1-r}\left(\int_{-\infty}^{\infty}\left|f^{\prime}(x)\right|^{p} d x\right)^{r / p}
$$

where $p=q r /(1-q+2 q r)$ for $p, q>1$. The sharp constant in (2.6) is given by

$$
K(q, r)=\left[\frac{q-1}{2 q r} Q\left(\frac{1}{q r}, \frac{q-q r-1}{q r}\right)\right]^{r}, \quad Q(x, y)=\frac{(x+y)^{-(x+y)} \Gamma(x+y)}{x^{-x} y^{-y} \Gamma(x) \Gamma(y)} .
$$

For (2.4), we take $r=1 / 2, p=2$, and $q=4$ to obtain the optimal value of the constant as $C^{1 / 4}=K(4,1 / 2)=\sqrt{3 /(2 \pi)}$ yielding the sharp inequality

$$
\int h^{4} d x \leqslant \frac{9}{4 \pi^{2}}\left(\int h d x\right)^{2} \int h_{x}^{2} d x
$$

and hence we obtain the value of the critical mass as

$$
M_{c}=2 \pi \sqrt{2 / 3} \approx 5.1302 .
$$

Now consider the solution $h(x, t)$ of $(1.1)$ on the interval $-1 \leqslant x \leqslant 1$ with periodic boundary conditions. We make use of an indirect argument based on the monotone decrease of energy (1.5) to show that blow-up is not possible below a certain mass.

Let the minimum of the solution at each time be achieved at the point $\underline{x}(t)$, that is $h(\underline{x})=h_{\min } \geqslant 0$, then define the compactly-supported function

$$
\underline{h}(x)= \begin{cases}h(x)-h_{\min } & \underline{x} \leqslant x \leqslant \underline{x}+2 \\ 0 & \text { otherwise on } \mathbb{R}\end{cases}
$$


Also, define the integrals

$$
\underline{I}_{q}=\int_{-\infty}^{\infty} \underline{h}^{q} d x, \quad \underline{D}_{2}=\int_{-\infty}^{\infty} \underline{h}_{x}^{2} d x,
$$

and

$$
\underline{M} \equiv \underline{I}_{1}=\int_{-\infty}^{\infty} \underline{h} d x .
$$

Note that $\underline{D}_{2}=\int_{-1}^{1} h_{x}^{2} d x$ and $\underline{M}=M-2 h_{\min }$ with $M \geqslant \underline{M} \geqslant 0$.

Using the above notation, the statement of decrease of the energy, $\mathscr{E}_{0} \geqslant \mathscr{E}(t)$ for $t \geqslant 0$, can then be written as

$$
12 \mathscr{E}_{0} \geqslant 6 \underline{D}_{2}-\int_{-1}^{1} h^{4} d x .
$$

We expand the integral on the right as

$$
\begin{aligned}
\int_{-1}^{1} h^{4} d x & =\int_{\underline{x}}^{\underline{x}+2}\left(\underline{h}+h_{\min }\right)^{4} d x \\
& =\underline{I}_{4}+4 h_{\min } \underline{I}_{3}+6 h_{\min }^{2} \underline{I}_{2}+4 h_{\min \underline{M}}^{3}+2 h_{\min }^{4}
\end{aligned}
$$

Since $\underline{h}(x)$ has been appropriately defined on $\mathbb{R}$, we can apply Sz.-Nagy's result (2.6) to yield

$$
\underline{I}_{q} \leqslant \mathscr{K}_{q} \underline{M}^{(q+2) / 3} \underline{D}_{2}^{(q-1) / 3}
$$

for $q>1$ with $\mathscr{K}_{q}=K(q, 2(q-1) /(3 q))$. Consequently (2.13) yields

$$
12 \mathscr{E}_{0} \geqslant\left(6-\mathscr{K}_{4} \underline{M}^{2}\right) \underline{D}_{2}-4 h_{\min } \mathscr{K}_{3} \underline{M}^{5 / 3} \underline{D}_{2}^{2 / 3}-6 h_{\min }^{2} \mathscr{K}_{2} \underline{M}^{4 / 3} \underline{D}_{2}^{1 / 3}-4 h_{\min }^{3} \underline{M}-2 h_{\min }^{4},
$$

and note that $\mathscr{K}_{4}=6 / M_{c}^{2}$. Suppose that blow-up were to occur at some (finite or infinite) time $t \rightarrow t_{c}$ with $\underline{D}_{2} \rightarrow \infty$. In this limit, the first term on the right side of (2.16) is the dominant term, $6\left(1-\underline{M}^{2} / M_{c}^{2}\right) \underline{D}_{2}$. If this term were positive then the decrease of energy would be eventually contradicted for $t$ sufficiently close to $t_{c}$. Therefore blow-up can not occur if $\underline{M}<M_{c}$. Consequently, since $h_{\min }(t) \geqslant 0$, this implies that on a periodic interval, solutions below the critical mass $M_{c}$ can not blow up.

Gagliardo-Nirenberg inequalities with optimal constants have also been used in connection with blow-up results for the nonlinear Schrodinger equation [28, 60] and for estimating decay rates to self-similar solutions of nonlinear diffusion equations [24].

\section{Dynamical framework: similarity variables}

We now make use of similarity solutions to describe the dominant dynamics of (1.1). As described by Barenblatt [3], self-similar solutions occur as intermediate asymptotic states in systems where, under appropriate rescalings of the dependent and independent variables, the structure of the solution remains unchanged as the system evolves towards a singular limit. Our analysis of this behavior in this fourth-order nonlinear PDE follows the techniques for studying similarity solutions in analogous second-order problems given in the works of Giga \& Kohn [29, 31, 32, 33, 34] and others [22, 30]. 
To begin, we derive the forms of the first-type self-similar solutions using dimensional analysis [3]. Consider rescaling the length-, height-, and time-scales of the solution according to

$$
x=\mathrm{L} \hat{x}, \quad t=\mathrm{T} \hat{t}, \quad h(x, t)=\mathrm{H} \hat{h}(\hat{x}, \hat{t}) .
$$

This change of variables applied to (1.1) yields

$$
\left[\frac{\mathrm{H}}{\mathrm{T}}\right] \frac{\partial \hat{h}}{\partial \hat{t}}=-\left[\frac{\mathrm{H}^{4}}{\mathrm{~L}^{2}}\right] \frac{\partial}{\partial \hat{x}}\left(\hat{h}^{3} \frac{\partial \hat{h}}{\partial \hat{x}}\right)-\left[\frac{\mathrm{H}^{2}}{\mathrm{~L}^{4}}\right] \frac{\partial}{\partial \hat{x}}\left(\hat{h} \frac{\partial^{3} \hat{h}}{\partial \hat{x}^{3}}\right) .
$$

If relations between $\mathrm{L}, \mathrm{T}, \mathrm{H}$ can be found that make (3.2) scale-invariant, then those relations correspond to self-similar solutions. Balancing the coefficients of the second-and fourth-order spatial operators determines that the scale of the film thickness is inversely proportional to the horizontal lengthscale, $\mathrm{H}=1 / \mathrm{L}$. A consequence of this relation is that similarity solutions will preserve their mass. Additionally balancing the time derivative term to obtain the distinguished limit yields the relation between the lengthscale and the timescale, $\mathrm{L}=\mathrm{T}^{1 / 5}$, and hence $\mathrm{H}=\mathrm{T}^{-1 / 5}$. These scalings yield two classes of similarity solutions:

(i) infinite-time spreading solutions: $\quad \mathrm{H} \rightarrow 0$ and $\mathrm{L} \rightarrow \infty$ as $\mathrm{T} \rightarrow \infty$,

(ii) finite-time blow-up solutions: $\quad \mathrm{H} \rightarrow \infty$ and $\mathrm{L} \rightarrow 0$ as $\mathrm{T} \rightarrow 0$.

The scaling analysis suggests a change of variables to similarity coordinates,

$$
h(x, t)=\frac{1}{\tau} H(\eta, s), \quad \eta=\frac{x-x_{c}}{\tau}, \quad s=-\frac{1}{\sigma} \ln \tau,
$$

where $\tau=\tau(t)$ and $\sigma$ is a constant. Note that all solutions of the original PDE can be represented in this form. Substituting this form into (1.3) yields a nonlinear separation of variables, where $H(\eta, s)$ satisfies the similarity $\mathrm{PDE}$

$$
\frac{\partial H}{\partial s}=-\frac{\partial}{\partial \eta}\left(H \frac{\partial}{\partial \eta}\left[\frac{1}{2} \sigma \eta^{2}+\frac{1}{3} H^{3}+H_{\eta \eta}\right]\right),
$$

and $\tau$ is the solution of the ordinary differential equation,

$$
\frac{d \tau}{d t}=-\sigma \tau^{-4} \quad \rightarrow \quad \tau=\left(5 \sigma\left[t_{c}-t\right]\right)^{1 / 5}
$$

Here $\sigma$ is a constant with $\sigma= \pm 1$ corresponding to the cases:

(i) $\quad \sigma=-1 \quad$ infinite-time spreading $(h \rightarrow 0$ as $t \rightarrow \infty) \quad$ for $t>t_{c}$,

(ii) $\quad \sigma=+1 \quad$ finite-time blow-up $\left(h \rightarrow \infty\right.$ as $\left.t \rightarrow t_{c}\right) \quad$ for $t<t_{c}$.

Note that $x_{c}$ and $t_{c}$ are constants corresponding to the spatial position and critical time associated with the similarity solution (3.3). Both limits, $t \rightarrow \infty$ in case (i), and $t \rightarrow t_{c}$ in case (ii), correspond to the limit $s \rightarrow \infty$ in the similarity variables. The variable $s$ gives the measure of time in (3.4). This formulation is very convenient for studying stability and rates of convergence to self-similar dynamics [20,33, 34]. 
Note that in addition to the cases given by (3.6), we can formally incorporate another class of behavior into the dynamics covered by (3.4):

(iii) $\quad \sigma=0 \quad$ near-equilibrium dynamics

for all $t$.

That is, for $\sigma=0$, the mapping $\{s \rightarrow t, \eta \rightarrow x, H \rightarrow h\}$ formally reduces (3.4) to (1.3). We will show that in all three cases, under appropriate conditions, stable 'generalized equilibria' are approached $H(\eta, s) \rightarrow \bar{H}(\eta)$ as $s \rightarrow \infty$. These are the stable steady-states for $\sigma=0$, and the stable self-similar solutions for $\sigma= \pm 1$. When necessary to avoid confusion, we will label these generalized equilibria as $\bar{H}^{\sigma}(\eta)$ (as in $\left.\bar{H}^{+}, \bar{H}^{-}, \bar{H}^{0}\right)$, otherwise we will suppress the $\sigma$ to avoid clutter. In fact there are many connections that we will explore between these different classes of solutions. While equation (3.4) is formally equivalent to the original equation (1.1) for any $\sigma$, different choices for $\sigma$ imply dramatically different dynamical behaviors of solutions. Studying the structure and stability of solutions of (3.4) will yield a better understanding of the possible dynamics in (1.1).

\subsection{Boundary conditions and general properties}

Equation (3.4) is a conservation law for $H, \partial_{s} H+\partial_{\eta} Q=0$, where the flux $Q=H \partial_{\eta} P$ is defined in terms of the generalized pressure,

$$
P=\frac{1}{2} \sigma \eta^{2}+\frac{1}{3} H^{3}+H_{\eta \eta}
$$

Consequently, the mass, which is scale-invariant,

$$
M \equiv \int H(\eta, s) d \eta=\int h(x, t) d x
$$

is conserved subject to appropriate no-flux boundary conditions. Two classes of such boundary conditions are relevant for the solutions of (1.1) that we study: (a) periodic boundary conditions on $-1 \leqslant x \leqslant 1$, and (b) no-flux conditions on the free-boundaries of compactly-supported solutions, $-L(s) \leqslant \eta \leqslant L(s)$.

Analysis of solutions of the general class of thin film equations (1.2) [16, 17] shows that compactly-supported solutions of (3.4) must satisfy the conditions

$$
H(L(s), s)=0, \quad H_{\eta}(L(s), s)=0 .
$$

That is, at the edge of the region of support (or interface) $\eta=L$, the solution must be tangent to the exterior trivial state $H \equiv 0$ for $|\eta|>L$. Requiring no-flux of mass across the interface yields a Rankine-Hugoniot type jump condition (see [52, 61] for example) for the motion of the interface,

$$
\frac{d L}{d s}=\frac{[Q]}{[H]}=\partial_{\eta} P(L(s), s) .
$$

Simplifying this general relation yields the evolution equation for the right interface position $L(s)$,

$$
\frac{d L}{d s}=\sigma L+H_{\eta \eta \eta}(L(s), s)
$$


with a similar equation for the leftmost point in the region of support of the solution. In terms of the original spatial variable, the position of the interface is given by $x-x_{c}=$ $\ell(t) \equiv L(s) \tau$.

There is also an analogue of the energy (1.5) for the similarity PDE,

$$
E=\int \frac{1}{2} H_{\eta}^{2}-\frac{1}{12} H^{4}-\frac{1}{2} \sigma \eta^{2} H d \eta
$$

This energy functional is monotone decreasing with the rate of dissipation given by

$$
\frac{d E}{d s}=-\int H\left(\partial_{\eta} P\right)^{2} d \eta \leqslant 0
$$

Similar energy integrals for the porous medium equation were considered by Newman [51] and Witelski \& Bernoff [64]. We note that the similarity energy (3.13) is not the same as the energy (1.5) written in similarity variables,

$$
\mathscr{E}=\frac{1}{\tau^{3}} \int \frac{1}{2} H_{\eta}^{2}-\frac{1}{12} H^{4} d \eta .
$$

That is, $E$ and $\tau^{3} \mathscr{E}$ differ by a term proportional to the second moment of the similarity solution, $\int \eta^{2} H d \eta$. Note that for self-similar solutions $E$ and $\tau^{3} \mathscr{E}$ are constants. In order that $\mathscr{E}(t)$ be monotone decreasing for self-similar solutions: (i) for $\sigma=-1$, since $\tau$ is increasing with time, the integral in (3.15) must be positive for spreading solutions, and (ii) for $\sigma=1$, since $\tau$ decreases to zero as the blow-up time is approached, the integral in (3.15) must be negative for blow-up solutions. The sign of the contribution from the second moment term in (3.13) is consistent with these observations and hence the two classes of similarity solutions (spreading and blow-up) have distinctly signed similarity energies, $E$ positive and negative respectively.

\section{Generalized equilibria of (3.4): steady states and self-similar solutions}

The steady states and self-similar solutions of (1.3) are given by extrema of the energy (3.13); they satisfy the zero-dissipation equality in (3.14). That is, generalized equilibria $H=\bar{H}(\eta)$ can either have a constant pressure $\bar{P}$ over the whole domain and satisfy

$$
\bar{H}^{\prime \prime}+\frac{1}{3} \bar{H}^{3}+\frac{1}{2} \sigma \eta^{2}=\bar{P},
$$

or they can be trivial, $\bar{H} \equiv 0$. There are also compactly-supported weak solutions, called "droplets", with constant pressures over their regions of support and $\bar{H} \equiv 0$ elsewhere, with conditions (3.10) at the interfaces.

Both periodic and compactly-supported solutions of (4.1) must satisfy the compatibility condition defining the average pressure,

$$
\bar{P}=\frac{1}{2 \bar{L}} \int_{-\bar{L}}^{\bar{L}} \frac{1}{3} \bar{H}^{3}+\frac{1}{2} \sigma \eta^{2} d \eta,
$$

where $\bar{L}$ is the half-length of the interval of support, with $\bar{L}=1$ for the periodic steadystates. The presence of the pressure in (4.1) with the compatibility condition (4.2), means 
that (4.1) is effectively a nonlocal problem [37]. For some of the follow analysis it is more convenient to write (4.1) as the equivalent third-order local problem

$$
\bar{H}^{\prime \prime \prime}+\bar{H}^{2} \bar{H}^{\prime}+\sigma \eta=0 .
$$

We consider even solutions of equation (4.1), these can be obtained from the solution of the associated Neumann problem for (4.1) or (4.3) on the half-interval $0 \leqslant \eta \leqslant \bar{L}$ with the boundary conditions,

$$
\bar{H}^{\prime}(0)=0, \quad \bar{H}^{\prime}(\bar{L})=0,
$$

where for compactly-supported solutions, we also impose $\bar{H}(\bar{L})=0$ at the interface. We address each of the three cases: $\sigma=0, \sigma=-1$, and $\sigma=1$ separately in the following sections.

\subsection{Classical steady states of the periodic problem, $\bar{h}(x)$}

We first consider the positive steady states $h=\bar{h}(x)$ of the periodic problem for (1.1) on $-1 \leqslant x \leqslant 1$. These solutions satisfy (4.3) with $\sigma=0$,

$$
\bar{h}^{\prime \prime \prime}+\bar{h}^{2} \bar{h}^{\prime}=0 \text {. }
$$

This equation is translation invariant, and apart from spatial translations, without loss of generality, imposing the Neumann conditions (4.4) on the half-interval $0 \leqslant x \leqslant 1$ yields all of the steady solutions. Having only two boundary conditions, this third-order problem is underspecified and admits multiple solutions. Indeed, constant states, $\bar{h}=\frac{1}{2} M$ for any positive mass $M>0$ constitutes a continuous one-parameter family of trivial solutions. At critical values of the mass, branches of nontrivial solutions bifurcate from this family. To obtain the form of these solutions, we can expand $\bar{h}$ as a perturbation series in the neighborhood of a bifurcation point, $M=M_{*}$,

$$
\bar{h}(x)=\frac{1}{2} M_{*}+\epsilon \bar{h}_{1}(x)+\epsilon^{2} \bar{h}_{2}(x)+\epsilon^{3} \bar{h}_{3}(x)+\cdots,
$$

where $0 \leqslant \epsilon \ll 1$. To keep the mass fixed (1.4), all of the perturbations $\bar{h}_{k}(x)$ must have zero mean. The bifurcation analysis then follows in a straightforward manner from substituting this expansion into (4.5) and solving the resulting regular perturbation problem [40, 41]. At $O(\epsilon)$ we get

$$
\bar{h}_{1}^{\prime \prime \prime}+\frac{1}{4} M_{*}^{2} \bar{h}_{1}^{\prime}=0 .
$$

Nontrivial solutions of this problem occur at the bifurcation points,

$$
M_{*}=2 n \pi, \quad \bar{h}_{1}(x)=A \cos (n \pi x),
$$

for $n=1,2,3, \cdots$. We focus our attention on the primary bifurcation point, $n=1$, $M_{1}=2 \pi$, since as will be shown, all of the higher-order bifurcations involve only unstable solutions; see Theorem 5 of Laugesen \& Pugh [42]. The higher-order branches of solutions correspond to rescaled periodic extensions of this fundamental branch. The reflection symmetry present in this problem forces the quadratic terms in the expansion to vanish and therefore it is necessary to expand $h$ to higher order, $O\left(\epsilon^{3}\right)$, to determine 

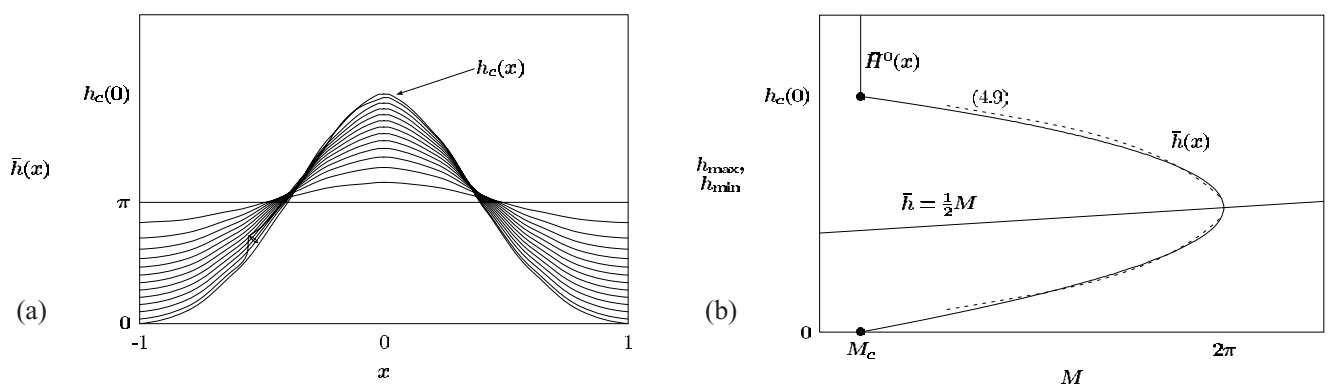

FIGURE 2. (a) The first branch of positive steady-state solutions, $\bar{h}(x)$ and the limiting compactlysupported solution $h_{c}(x)$, and (b) the bifurcation diagram for $h_{\max }, h_{\min }$ in terms of the mass.

a relation between $\epsilon$ and the amplitude $A$. Following the method described in Bertozzi et al. [14], at the first bifurcation point, the local structure for $M \leqslant 2 \pi$ is given by

$$
\bar{h}(x) \sim \frac{1}{2} M+A \cos (\pi x), \quad A^{2}=\frac{12}{5} \pi(2 \pi-M) .
$$

Having obtained the steady states, their corresponding pressure and energy can be found via quadrature,

$$
\bar{p}=\int_{-1}^{1} \frac{1}{3} \bar{h}^{3} d x>0, \quad \mathscr{E}=\int_{-1}^{1} \frac{1}{2} \bar{h}_{x}^{2}-\frac{1}{12} \bar{h}^{4} d x \leqslant 0 .
$$

Much more thorough results on the steady states of the generalized thin film equation (1.2) are given in the works of Laugesen \& Pugh [42, 43, 44, 45].

We plot the bifurcation diagram for these steady state solutions in terms of the maximum and minimum of the solution $h_{\max }, h_{\min }$, as a function of the mass (see Figure $2 \mathrm{~b}$ ). Note that there is a critical value of the mass, $M=M_{c}$, where the minimum of $\bar{h}(x)$ crosses through zero. At this point, the branch of positive solutions ends. Next, we turn to the family of compactly-supported solutions that begins from that point on the bifurcation diagram, $M=M_{c}$.

\subsection{Compactly-supported steady state solutions, $\bar{H}^{0}(x)$}

Above, we parametrized the solutions in terms of their mass, we now show that this is not appropriate for the study of the compactly-supported steady states. Instead, we obtain the solutions in terms of their pressures $\bar{p}$ from the appropriate form of (4.1) with $\sigma=0$,

$$
\bar{h}_{x x}+\frac{1}{3} \bar{h}^{3}=\bar{p} .
$$

To calculate this branch of solutions, we consider the solution $\bar{h}=h_{c}(x)$ with support on the half-interval $0 \leqslant x \leqslant 1$ satisfying the boundary conditions

$$
h_{c}^{\prime}(0)=0, \quad h_{c}(1)=0, \quad h_{c}^{\prime}(1)=0 .
$$


Integrating (4.11), this solution satisfies the first-order equation

$$
\left(\frac{d h_{c}}{d x}\right)^{2}=\frac{1}{6} h_{c}\left(h_{c}^{3}(0)-h_{c}^{3}\right)
$$

where the boundary conditions determine the pressure to be $\bar{p}=h_{c}^{3}(0) / 12$. Consequently, straightforward calculations yield the maximum value of the solution,

$$
h_{c}(0)=\int_{0}^{1} \frac{\sqrt{6} d y}{\sqrt{y\left(1-y^{3}\right)}}=\frac{2 \pi \sqrt{2 \pi / 3}}{\Gamma(5 / 6) \Gamma(2 / 3)} \approx 5.9490,
$$

and the mass of the solution,

$$
M_{c}=2 \int_{0}^{1} \frac{\sqrt{6} y d y}{\sqrt{y\left(1-y^{3}\right)}}=2 \pi \sqrt{2 / 3} .
$$

Significantly, this critical mass is identical to the critical mass in the criterion for blow-up, (2.9). A further calculation using $\int_{0}^{1} y^{4} / \sqrt{y-y^{4}} d y=\pi / 6$, shows that the energy of this solution is $\mathscr{E}=0$. Indeed, this smooth, non-negative solution $h_{c}(x)$ is the limit of the set of solutions $\bar{h}(x)$ as $M \rightarrow M_{c}$ (see Figure 2a).

Note that the problem specifying $h_{c}(x)$ on $-1 \leqslant x \leqslant 1,(4.12,4.13)$, is invariant under changes of the lengthscale, and defines a compactly-supported solution for any $\bar{L}>0$ given by

$$
\bar{H}^{0}(x)=\frac{1}{\bar{L}} h_{c}(x / \bar{L}), \quad|x| \leqslant \bar{L} .
$$

The mass of each member of this family of solutions is $M_{c}$, independent of $\bar{L}$, while the pressure scales as $\bar{p}=h_{c}^{3}(0) /\left(12 \bar{L}^{3}\right)$ where $h_{\max }=h_{c}(0) / \bar{L}$. For the boundary value problem for (1.1) on $-1 \leqslant x \leqslant 1$, the range of $\bar{L}$ is restricted to $0 \leqslant \bar{L} \leqslant 1$. This branch of solutions, with $h_{\min }=0$ and $h_{\max }=h_{c}(0) / \bar{L}$ for $\bar{L} \leqslant 1$ is indicated in Figure $2 \mathrm{~b}$. We note that these steady-state solutions trivially satisfy the no-flux interface condition (3.12) for $\sigma=0$ since from $(4.5), h_{c}^{\prime \prime \prime}(1)=0$, and hence the interface position is indeed fixed.

Within its interval of support, the steady droplet solution $\bar{H}^{0}(x)$ is one period of a cnoidal wave. There are also branches of compactly-supported equilibria that bifurcate from the higher-order branches of positive solutions. These compactly-supported solutions contain multiple copies of the fundamental compact solution $h_{c}(x)$. However, these solutions do not necessarily have to be periodic extensions of $h_{c}(x)$. As long as the support of all of the droplets remains disjoint, each droplet is independent of the others - hence the droplets can be non-uniformly spaced and also have different values of $\bar{h}_{\text {max }}$ (though the mass of each drop is fixed to be $M_{c}$ ). There is an uncountable multiplicity of such weak steady state solutions, also called 'droplet configurations' by Laugesen \& Pugh [42, 45]. Later we will show that these states are very sensitive to small perturbations of the mass.

\subsection{Infinite-time self-similar spreading solutions, $\bar{H}^{-}(\eta)$}

While for $\sigma=0$ branches of both positive solutions and compactly-support steady states exist, for $\sigma= \pm 1$ only compactly-supported, non-negative, finite-mass, self-similar 


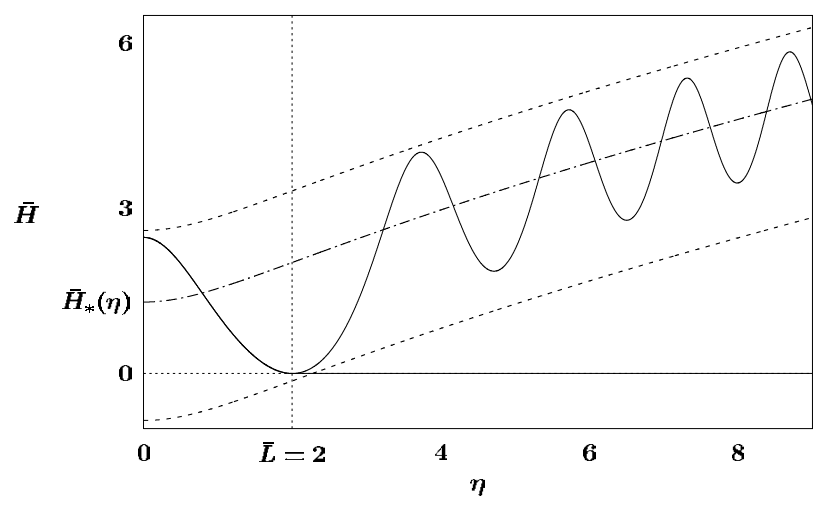

FIGURE 3. The classical solution of (4.1) with $\sigma=-1$ and $\bar{L}=2$ is bounded within a fixed-width strip about $\bar{H}_{*}(\eta)=\left(3\left[\bar{P}+\frac{1}{2} \eta^{2}\right]\right)^{1 / 3}$ for $\eta \geqslant 0$ with oscillations about $\bar{H}_{*}$ of decreasing amplitude. This construction demonstrates that no 'multi-bump' compact, non-negative spreading self-similar solutions $\bar{H}^{-}(\eta)$ are possible.

solutions exist, $\bar{H}^{ \pm}(\eta)$. We begin describing the structure of these solutions by demonstrating that there is only a single branch of symmetric spreading similarity solutions, parametrized by their mass, for $\sigma=-1$. We accomplish this by making use of a dissipated quantity for solutions of (4.1).

Solutions of the steady-state problem, (4.1) with $\sigma=0$, have the conserved quantity (a Hamiltonian for the ODE),

$$
K=\frac{1}{2} \bar{H}_{\eta}^{2}+\frac{1}{12} \bar{H}^{4}-\frac{1}{3} \bar{H}_{*}^{3} \bar{H}
$$

where $\bar{H}_{*}=(3 \bar{P})^{1 / 3}$ gives the position of an elliptic fixed point in the phase plane representation of (4.1). That is, all solutions of (4.1) are oscillations about $\bar{H}_{*}$. For selfsimilar spreading solutions, satisfying (4.1) with $\sigma=-1$, we define $\bar{H}_{*}=\left(3\left[\bar{P}+\frac{1}{2} \eta^{2}\right]\right)^{1 / 3}$ by analogy. With this definition, $K,(4.17)$ is a monotone decreasing quantity for solutions of (4.1) with $\sigma=-1$,

$$
\frac{d K}{d \eta}=-\eta \bar{H} \leqslant 0 .
$$

Interpreting (4.1) as a slowly-varying phase plane system for $\eta^{2} \ll \bar{P}$, equation (4.18) implies that the solutions are decreasing-amplitude oscillations about $\bar{H}_{*}$, see Figure 3. Since $\bar{H}_{*}(\eta)$ is an increasing function, if $\bar{H}(\eta)$ has a minimum at $\bar{H}(\bar{L})=0$, then other minima for $\eta>\bar{L}$ must have $\bar{H}>0$ (and similarly, other minima for $\eta<\bar{L}$ must have $\bar{H}<0$ ). Therefore, the only family of symmetric non-negative compactly-supported spreading solutions possible is the one with monotone decreasing solutions on $0 \leqslant \eta \leqslant \bar{L}$. A rigorous proof that the solutions must be monotone decreasing was given by Beretta [5] (Lemma 2.5).

This family of similarity solutions can be described in terms of their mass (see Figure 5), but it is more conveniently studied by considering the dependence of the solutions on $\bar{L}$ (see Figure 4). To describe this branch of similarity solutions, we consider asymptotic limits of the solutions of (4.3) when the interval of support vanishes, $\bar{L} \rightarrow 0$. Begin by 


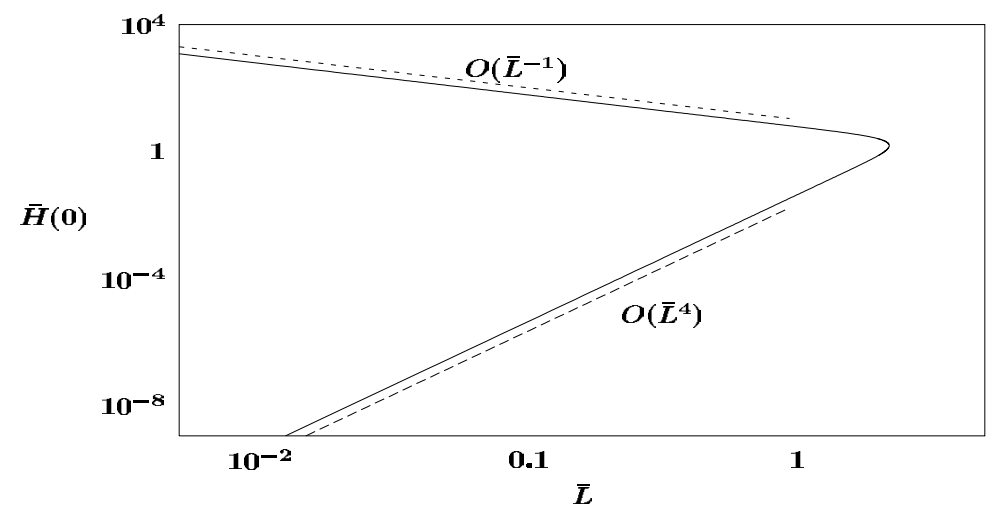

FIGURE 4. The continuous family of infinite-time spreading similarity solutions, $\bar{H}^{-}(\eta)$, shown parametrized by maximum height versus length of the region of support.

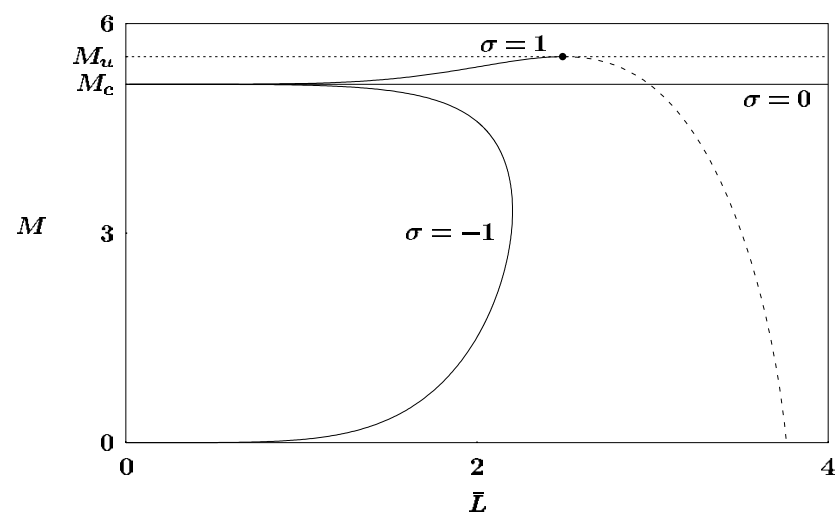

FIGURE 5. The three classes of generalized equilibria (steady-state, spreading, and blow-up solutions) shown parametrized by their mass vs. length of the region of support.

rescaling $\eta=\bar{L} z$, so $0 \leqslant z \leqslant 1$. Then one distinguished limit of (4.3) is the case of solutions with vanishingly small masses, $\bar{H}(\eta)=\bar{L}^{4} \mathscr{H}(z)$, satisfying the equation

$$
\mathscr{H}^{\prime \prime \prime}+\sigma z=-\bar{L}^{10} \mathscr{H}^{2} \mathscr{H}^{\prime} .
$$

The solution can then be written as a regular perturbation series, $\mathscr{H}(z)=\mathscr{H}_{0}(z)+$ $\bar{L}^{10} \mathscr{H}_{1}(z)+\cdots$. Solving to leading order yields,

$$
\bar{H}(\eta)=\frac{1}{24}\left(\bar{L}^{2}-\eta^{2}\right)_{+}^{2}+O\left(\bar{L}^{14}\right), \quad \bar{L} \rightarrow 0,
$$

with the mass $M \sim 2 \bar{L}^{5} / 45$. This is the source-type similarity solution of the thin film equation $h_{t}=-\left(h h_{x x x}\right)_{x}$ [10]. In Figure 4 , this limiting behavior of the set of solutions is illustrated in terms of the value of the maximum of the solution plotted against $\bar{L}$, $\bar{H}(0) \sim \bar{L}^{4} / 24$. 


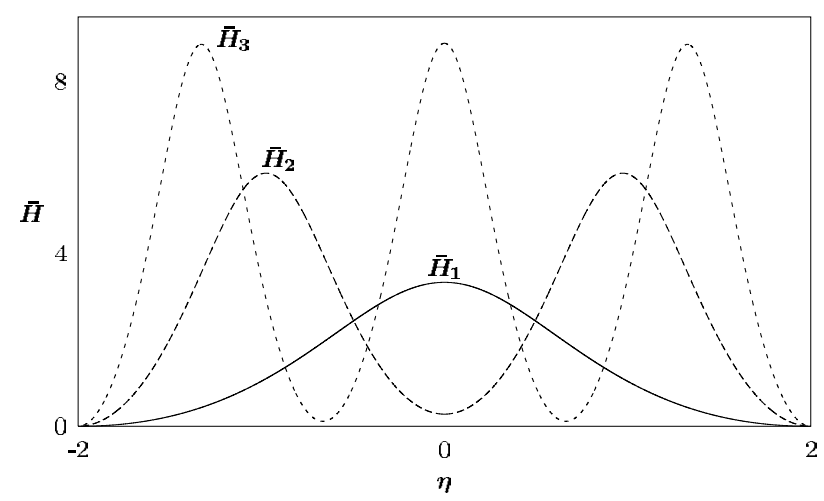

FigURE 6. The first three blow-up similarity solutions for $\bar{L}=2$.

The other distinguished limit of (4.3) for $\bar{L} \rightarrow 0$ describes finite mass solutions given by the scalings $\eta=\bar{L} z$ and $\bar{H}(\eta)=\mathscr{H}(z) / \bar{L}$,

$$
\mathscr{H}^{\prime \prime \prime}+\mathscr{H}^{2} \mathscr{H}^{\prime}=\bar{L}^{5} z
$$

The solution can then be written as a regular perturbation series of the form $\mathscr{H}(z)=$ $\mathscr{H}_{0}(z)+L^{5} \mathscr{H}_{1}(z)+\cdots$. Recalling that $h_{c}(x)$ is the compact solution of (4.5), we find that the leading order solution of (4.21) is the compactly-supported steady state, $\mathscr{H}_{0}=h_{c}(z)$. Hence, in this limit, the spreading similarity solutions are given by

$$
\bar{H}(\eta)=\frac{1}{\bar{L}} h_{c}(\eta / \bar{L})+O\left(\bar{L}^{4}\right), \quad \bar{L} \rightarrow 0,
$$

with mass $M \rightarrow M_{c}$ as $\bar{L} \rightarrow 0$. We will return to examine this limit further in the next section. In Figure 4 this limiting behavior yields $\bar{H}(0) \sim h_{c}(0) / \bar{L}$. In Figure 5 , this branch of solutions is represented in terms of its mass plotted against its interval of support.

This problem for spreading self-similar solutions of (1.1) was considered by Beretta [5]. Although her paper claims to prove existence of self-similar solutions for all positive mass, this is not the case [6]. Our numerical calculations show that spreading self-similar solutions exist only for the range of masses $0 \leqslant M \leqslant M_{c}$, see Figure 5 .

\subsection{Finite-time blow-up self-similar solutions, $\bar{H}^{+}(\eta)$}

In contrast to the spreading similarity solutions, for the blow-up solutions there is no argument to eliminate the possibility of non-negative multi-bump blow-up solutions, and such solutions do exist, see Figure 6. In fact, continuous branches of multi-bump solutions $\bar{H}_{n}^{+}(\eta)$ exist, parametrized by their interval of support (see Figure 8 ). We shall now describe the structure of these solution branches.

To see that for fixed $\bar{L}$, as the number of bumps in the solutions increases, the solutions are nearly periodic oscillations, consider the rescaling for an $n$-bump solution,

$$
\bar{H}(\eta)=n \mathscr{H}(z), \quad \eta=\frac{z}{n},
$$


(a)

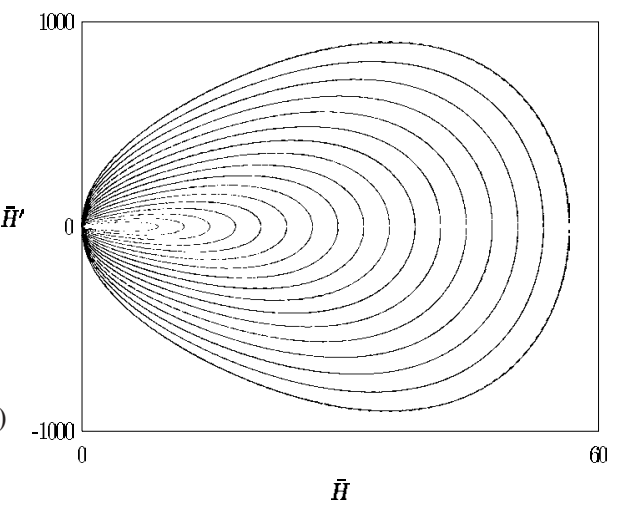

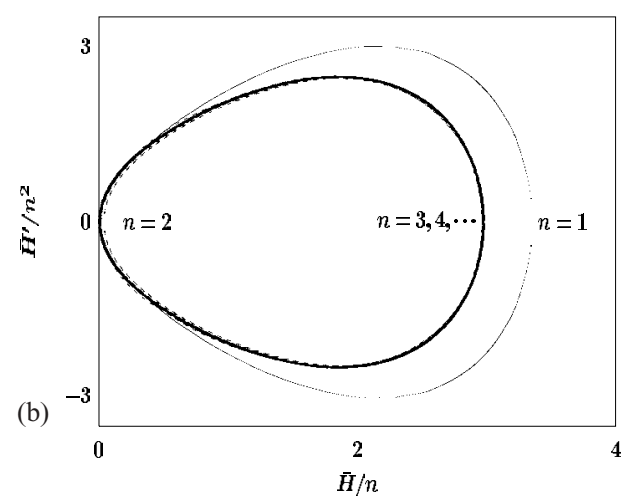

FIgURE 7. (a) A representation of the first twenty multi-bump blow-up solutions for $\bar{L}=2$ in the $\bar{H}, \bar{H}^{\prime}$ phase plane. (b) The same solutions plotted in the rescaled phase plane suggested by (4.23).
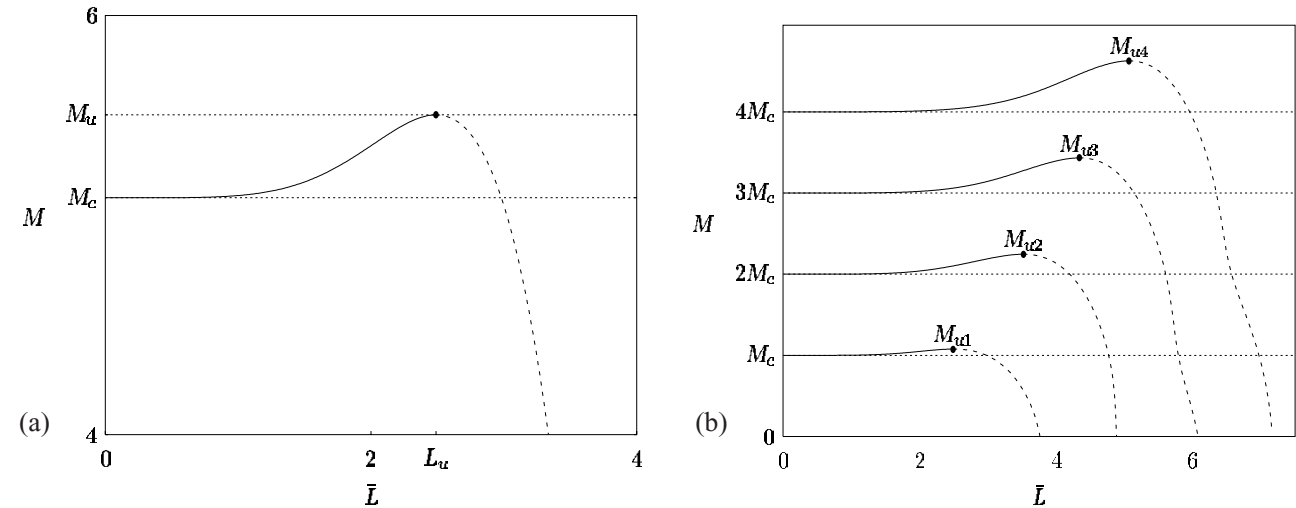

FIGURE 8. (a) The first branch of self-similar blow-up solutions. Non-negative solutions (solid curve) exist with masses in the range $M_{c}<M \leqslant M_{u}$. The dashed curve for $\bar{L}>L_{u}$ shows a continuation of the family where the solutions become negative over some interval. (b) The first four branches of finite-time blow-up solutions.

then (4.3) with $\sigma=1$ becomes

$$
\mathscr{H}^{\prime \prime \prime}+\mathscr{H}^{2} \mathscr{H}^{\prime}=-\frac{1}{n^{3}} z .
$$

For $0 \leqslant \eta \leqslant \bar{L}, z \leqslant O(n)$ and hence this is a regular perturbation as $n \rightarrow \infty$. Consequently, in this limit the blow-up solutions are given by the periodic steady states with slowly growing perturbations,

$$
\bar{H}_{n}^{+}(\eta)=n \bar{h}(n \eta)+O\left(n^{-2}\right)
$$

In contrast to the results for the $\bar{H}^{-}(\eta)$, the amplitude of the oscillations in these solutions increases as $\eta$ increases allowing for multi-bump non-negative solutions. A validation of this asymptotic representation of the solutions for $n=3,4, \cdots$ is shown by the collapse of orbits corresponding to the first twenty multi-bump blow-up solutions in a scaled phase-plane plot (see Figure 7b). 


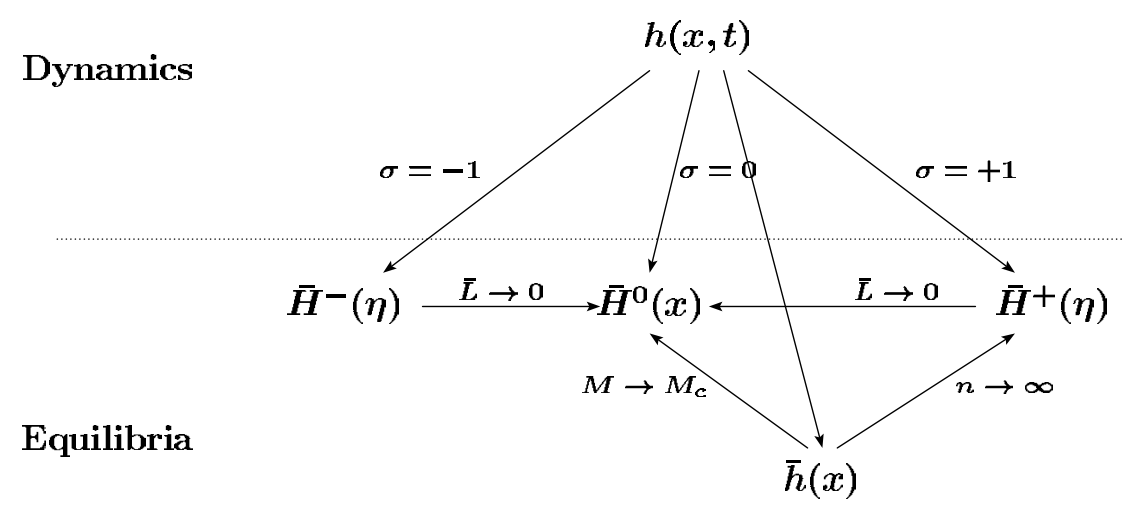

FIGURE 9. A schematic diagram representing: (i) the relations between the generalized equilibria studied in $\S 4$, and (ii) the stability and dynamics studied in $\S 5$, for different values of $\sigma$.

\subsubsection{Asymptotics for $\bar{L} \rightarrow 0$}

To examine the solution branch for $\bar{L} \rightarrow 0$, we return to the scalings $\eta=\bar{L} z, \bar{H}(\eta)=$ $\mathscr{H}(z) / \bar{L}$, used for (4.21). This choice of variables transforms (4.3) to

$$
\mathscr{H}^{\prime \prime \prime}+\mathscr{H}^{2} \mathscr{H}^{\prime}=-\sigma \bar{L}^{5} z
$$

with boundary conditions $\mathscr{H}(0)=\mathscr{H}(1)=\mathscr{H}^{\prime}(1)=0$. Note that as $\bar{L} \rightarrow 0$, (4.26) is a regular perturbation of the steady problem (4.5), which suggests the expansion

$$
\mathscr{H}(z)=h_{c}(z)+\sigma \bar{L}^{5} \mathscr{H}_{1}(z)+O\left(\bar{L}^{10}\right) .
$$

In terms of this rescaled solution, the mass is given by

$$
M=2 \int_{0}^{1} \mathscr{H}(z) d z=M_{c}+\sigma \bar{L}^{5} M_{1}+O\left(\bar{L}^{10}\right) .
$$

We obtain $M_{1}$ from the solution of the order $O\left(\bar{L}^{5}\right)$ problem for (4.26),

$$
\mathscr{H}_{1}^{\prime \prime \prime}+\left(h_{c}^{2}(z) \mathscr{H}_{1}\right)^{\prime}=-\sigma z .
$$

Integrating this equation once, then taking the inner product with $\left[z h_{c}(z)\right]^{\prime}$ (the null-mode associated with rescaling, see $\S 5$ ) yields a solvability condition to determine $M_{1}$,

$$
M_{1}=\frac{8}{h_{c}^{3}(0)} \int_{0}^{1} z^{2} h_{c}(z) d z \approx 1.083 \times 10^{-2} .
$$

Equation (4.28) describes the local structure of Figure 5 near $M=M_{c}$ for $\bar{L} \rightarrow 0$. It applies to both spreading and blow-up solutions $(\sigma= \pm 1)$ and also trivially to the compactly supported steady state $(\sigma=0)$. Consequently, this equation describes one aspect of the connection between the three families of generalized equilibria for $\bar{L} \rightarrow 0$ (see Figure 9).

Working similarly and using the nearly periodic structure of the higher-order blow-up solutions (4.25), we find that the mass on the $n^{\text {th }}$ branch takes the form

$$
M \sim n M_{c}+M_{n} \bar{L}^{5}, \quad \bar{L} \rightarrow 0,
$$


(see Figure $8 \mathrm{~b}$ ), where for $n=1,2, \cdots$,

$$
M_{n}=\frac{8}{n^{2} h_{c}^{3}(0)} \int_{0}^{1} z^{2} \bar{h}_{c}(n z+(n+1) \bmod 2) d z,
$$

with $\bar{h}_{c}(z)$ being the periodic extension of $h_{c}(z)$.

\subsubsection{The maximum mass point on the branch of blow-up solutions}

A striking feature of the branch of blow-up solutions represented in Figure 5 is the existence of a maximum value for the masses of the similarity solutions,

$$
M_{u} \approx 5.5258
$$

This is also shown in Figure 8a, where we clearly see that the mass increases monotonically with the length of support, up to the maximum mass $M_{u}$ at $\bar{L}=L_{u}$. The branch of solutions of (4.3) with $\sigma=1$ continues past this point, but for $\bar{L}>L_{u}$ the solutions have decreasing mass and do not satisfy the requirement of being non-negative (hence this part of the branch is shown with a dashed curve). In fact, all of the branches of blow-up solutions exhibit this structure - see Figure $8 \mathrm{~b}$ (where $M_{u}$ is denoted by $M_{u 1}$ for the first branch for solutions). From the boundary conditions at the interface, the solutions must be locally quadratic there,

$$
\bar{H}(\eta) \sim \frac{1}{2} \bar{H}^{\prime \prime}(\bar{L})(\bar{L}-\eta)^{2}, \quad \eta \rightarrow \bar{L}^{-} .
$$

Locally, the sign of the solution is given by the sign of $\bar{H}^{\prime \prime}(\bar{L})$. Consequently, the change of sign of the solutions at the maximum mass point implies that $\bar{H}^{\prime \prime}\left(L_{u}\right)=0$. We can achieve some understanding of why this occurs by examining how the solutions change with $\bar{L}$.

Consider the solutions near the one at the maximum mass point, $\bar{H}_{u}(\eta)$ with $\bar{L}=L_{u}$, with $\epsilon \equiv \bar{L}-L_{u} \rightarrow 0$,

$$
\bar{H}(\eta)=\bar{H}_{u}(\eta)+\epsilon H_{1}(\eta)+\cdots, \quad \bar{L}=L_{u}+\epsilon,
$$

where $\bar{H}_{u}$ satisfies (4.3), (4.4) and $\bar{H}_{u}\left(L_{u}\right)=0$. Correspondingly, the mass is given by $M=M_{u}+\epsilon M_{1}+O\left(\epsilon^{2}\right)$. Linearizing (4.3) about $\bar{H}_{u}$ for $\epsilon \rightarrow 0$ yields the problem for $H_{1}$,

$$
H_{1}^{\prime \prime \prime}+\left(\bar{H}_{u}^{2} H_{1}\right)^{\prime}=0 \quad 0 \leqslant \eta \leqslant L_{u},
$$

with the linearized boundary conditions,

$$
H_{1}(0)=0, \quad H_{1}\left(L_{u}\right)=0, \quad H_{1}^{\prime}\left(L_{u}\right)=-\bar{H}_{u}^{\prime \prime}\left(L_{u}\right)
$$

If $\bar{H}_{u}^{\prime \prime}\left(L_{u}\right)=0$ then this problem has the trivial solution and $M_{1}=0$ corresponding to a local extrema of the mass. The local analysis for $M_{u, n}$ on the higher-order branches follows identically. 


\section{Stability analysis}

Having catalogued all of the steady states and similarity solutions of (1.1) in the previous section, we must now determine which of these solutions are preferred by the dynamics of the PDE. That is, starting from generic initial data, are the solutions stable? The linearized analysis of the asymptotic stability of the generalized equilibria of (3.4) as $s \rightarrow \infty$ generally proceeds the same way in the three cases; $\sigma=-1,0,1$. However there are also notable differences in these cases since the results describe three qualitatively different behaviors; infinite-time spreading, infinite-time convergence to a steady state, and finite-time singularity formation.

There are several classes of perturbations that must be considered and distinguished to evaluate stability of the solutions of (1.1),

Perturbations of:

(a) the time of the singularity, $t_{c} \rightarrow t_{c}+\epsilon$,

(b) the position of the singularity, $x_{c} \rightarrow x_{c}+\epsilon$,

(c) the choice of discrete branches of self-similar solutions, $\bar{H}_{n}^{\sigma}(\eta) \rightarrow \bar{H}_{m}^{\sigma}(\eta)$,

(d) the mass of the self-similar solution, $M \rightarrow M+\epsilon$,

(e) the regime of dynamics selected, $\sigma_{1} \rightarrow \sigma_{2}$.

We discuss these possible instabilities in the framework of linear stability analysis of the self-similar solutions. Though, for the last two items, we will find that linear analysis is insufficient to fully describe the behavior and we shall employ numerics to illustrate the problems in the final section of this article.

While the study of the stability of self-similar processes is a complicated problem with respect to the original PDE (1.1), the use of similarity variables (3.3) considerably simplifies the difficulties. With respect to the similarity PDE (3.4), the self-similar solutions $\bar{H}^{\sigma}(\eta)$ are steady state solutions, hence classical linear stability theory can be applied to the problem in similarity coordinates $[22,32]$. Consider an infinitesimal perturbation to a generalized equilibrium solution of the form

$$
H(\eta, s)=\bar{H}(\eta)+\epsilon \hat{H}(\eta) e^{\lambda s},
$$

as $s \rightarrow \infty$ with $\epsilon \rightarrow 0$. Substituting (5.2) into (3.4), and linearizing for $\epsilon \rightarrow 0$ yields

$$
\lambda \hat{H}=\mathscr{L}(\bar{H}) \hat{H},
$$

where the linear operator $\mathscr{L}$ is defined by

$$
\mathscr{L}(\bar{H}) \hat{H} \equiv-\partial_{\eta}\left[\bar{H} \partial_{\eta}\left(\bar{H}^{2}+\partial_{\eta \eta}\right)\right] \hat{H} .
$$

Before considering the problem of obtaining the spectrum of (5.4), we address the eigenmodes that can be determined from symmetry considerations for (1.1).

In $\S 3$ we used the scale-invariance of (1.1) to determine the form of similarity solutions; now we use the equation's translation invariance in space and time to find eigenmodes associated with the actions of these symmetries. These symmetries generate continuous families of solutions, parametrized by their spatial position and time-shifts. In writing 
the variables to describe similarity solutions $(3.3,3.5)$, it is assumed that the position $x_{c}$ and time-shift $t_{c}$ of the solution are known exactly. In general, this is not the case, and perturbations in the values assumed for $\left(x_{c}, t_{c}\right)$ lead to instabilities associated with (5.1ab). This result can be demonstrated using the invariance of the PDE (1.1) with respect to spatial translations and time-shifts of the similarity solutions. Consider (5.1a), that is, suppose that there is a perturbation in the value of the time-shift, $t_{c} \rightarrow t_{c}+\epsilon$. This corresponds to a transformation from one solution of (1.1), to a time-shifted version, $h(x, t) \rightarrow h(x, t-\epsilon)$, which is also a solution of the PDE. Translating this shift into similarity variables, $\tau \rightarrow \tau\left(1+5 \epsilon \sigma e^{5 \sigma s}\right)^{1 / 5}$, and applying it to the generalized equilibrium $\bar{H}(\eta)$ yields

$$
\bar{H}(\eta) \rightarrow H(\eta, s)=\left(1+5 \epsilon \sigma e^{5 \sigma s}\right)^{-1 / 5} \bar{H}\left(\eta\left[1+5 \epsilon \sigma e^{5 \sigma s}\right]^{-1 / 5}\right) .
$$

Linearizing the action of this symmetry transformation for $\epsilon \rightarrow 0$, we can identify the eigenmode connected with time-shifts of the self-similar solution $\bar{H}(\eta)$,

$$
H(\eta, s) \sim \bar{H}(\eta)+\epsilon \hat{H}_{T}(\eta) e^{\lambda_{T} s}, \quad \hat{H}_{T}(\eta)=-\sigma \frac{d}{d \eta}(\eta \bar{H}), \quad \lambda_{T}=5 \sigma .
$$

A similar description applies to perturbations in the assumed position of the singularity, $x_{c} \rightarrow x_{c}+\epsilon,(5.1 \mathrm{~b})$. This case corresponds to spatial shifts of solutions of (1.1), $h(x, t) \rightarrow$ $h(x-\epsilon, t)$, and in terms of similarity variables,

$$
\bar{H}(\eta) \rightarrow H(\eta, s)=\bar{H}\left(\eta+\epsilon e^{\sigma s}\right) .
$$

Linearizing the action of this symmetry for infinitesimal spatial translations of the similarity solution $\bar{H}(\eta)$ yields the eigenmode,

$$
H(\eta, s) \sim \bar{H}(\eta)+\epsilon \hat{H}_{X}(\eta) e^{\lambda_{X} s}, \quad \hat{H}_{X}(\eta)=\bar{H}^{\prime}(\eta), \quad \lambda_{X}=\sigma .
$$

Since there are continuous families of similarity solutions, parametrized by their mass, it is also important to consider the influence of perturbations which could yield infinitesimal changes to the mass, $M \rightarrow M+\epsilon$, (5.1d). Apart from such perturbations, mass is conserved, so it can be argued that such perturbations must be neutrally stable,

$$
H(\eta, s) \sim \bar{H}(\eta)+\epsilon \hat{H}_{M}(\eta) e^{\lambda_{M} s}, \quad \hat{H}_{M}(\eta)=\frac{\partial \bar{H}}{\partial M}, \quad \lambda_{M}=0 .
$$

Having accounted for possible instabilities due to the definitions of the similarity variables and continuous symmetries of the PDE, we turn to the question of stability of $\bar{H}(\eta)$ with respect to perturbations that change the profile of the solutions, (5.1c), for the cases $\sigma=0,-1,1$.

\subsection{Periodic steady state solutions, $\bar{h}(x)$}

The problem for the linear stability of the periodic steady state solutions is obtained by formally replacing $(H, \eta, s) \rightarrow(h, x, t)$ in $(5.2,5.4)$ on the periodic domain $-1 \leqslant x \leqslant 1$. The linear stability of the constant solutions, $\bar{h}(x)=M / 2$, can be found explicitly by 


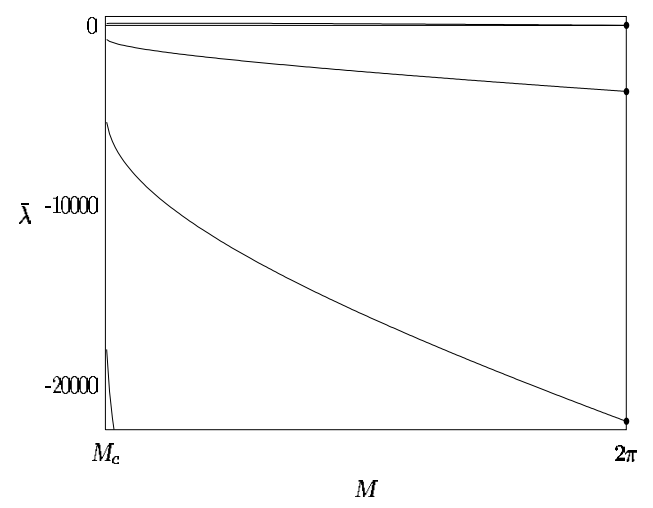

FIGURE 10. Eigenvalues for the linear stability of the first branch of periodic steady-state solutions. Solid dots for $M=2 \pi$ are given by (5.10).

substituting $h(x, t)=M / 2+\epsilon e^{i k \pi x} e^{\bar{\lambda} t}$ into (1.1) and linearizing,

$$
\bar{\lambda}_{k}=\frac{1}{8} M \pi^{2} k^{2}\left(M^{2}-4 \pi^{2} k^{2}\right), \quad k=0,1,2,3, \cdots .
$$

In particular, the constant solutions are stable for $M<2 \pi$ and unstable for $M>2 \pi$. Consequently, the bifurcation point shown in Figure $2 b$ is a supercritical bifurcation. Indeed, numerical calculation of the linear stability of the first branch of non-uniform steady states, $\bar{h}(x)$, shows that they are all unstable with a single positive eigenvalue. Additionally, solutions have a zero eigenvalue, $\lambda_{M}=0$, corresponding to infinitesimal perturbations of the mass for all $M$, as with $k=0$ in (5.10). There is also another zero eigenvalue, $\lambda_{X}=0$ for all $M$, corresponding to the translation invariance of the solutions on the periodic domain (this is a trivial mode for the branch of constant solutions). Figure 10 shows that the eigenvalues of this branch of solutions varies continuously with $M$ starting from the bifurcation point $M=2 \pi$ down to the end of the branch at $M \rightarrow M_{c}$. More thorough results on the linear stability of solutions of (1.2) can be found in Laugesen \& Pugh [42].

\subsection{The interface conditions for compactly-supported solutions}

To study the stability of compactly-supported solutions, we must allow for the possibility that both the profile and the region of support can evolve,

$$
H(\eta, s)=\bar{H}(\eta)+\epsilon \hat{H}(\eta) e^{\lambda s}, \quad L(s)=\bar{L}+\epsilon \hat{L} e^{\lambda s},
$$

and both symmetric and anti-symmetric perturbation modes are possible. As described above, $\hat{H}(\eta)$ satisfies (5.3) on $-\bar{L} \leqslant \eta \leqslant \bar{L}$. At the interface, we impose the linearized version of the interface conditions (3.10), i.e.

$$
\hat{H}(\bar{L})=0, \quad \hat{H}^{\prime}(\bar{L})+\hat{L} \bar{H}^{\prime \prime}(\bar{L})=0 .
$$

Linearizing the evolution equation for the interface (3.12) yields $\lambda \hat{L}=\sigma \hat{L}+\bar{H}^{\prime \prime \prime \prime \prime}(\bar{L}) \hat{L}+$ $\hat{H}^{\prime \prime \prime}(\bar{L})$. We can simplify this equation by differentiating (4.3) and evaluating it at $\eta=\bar{L}$ 

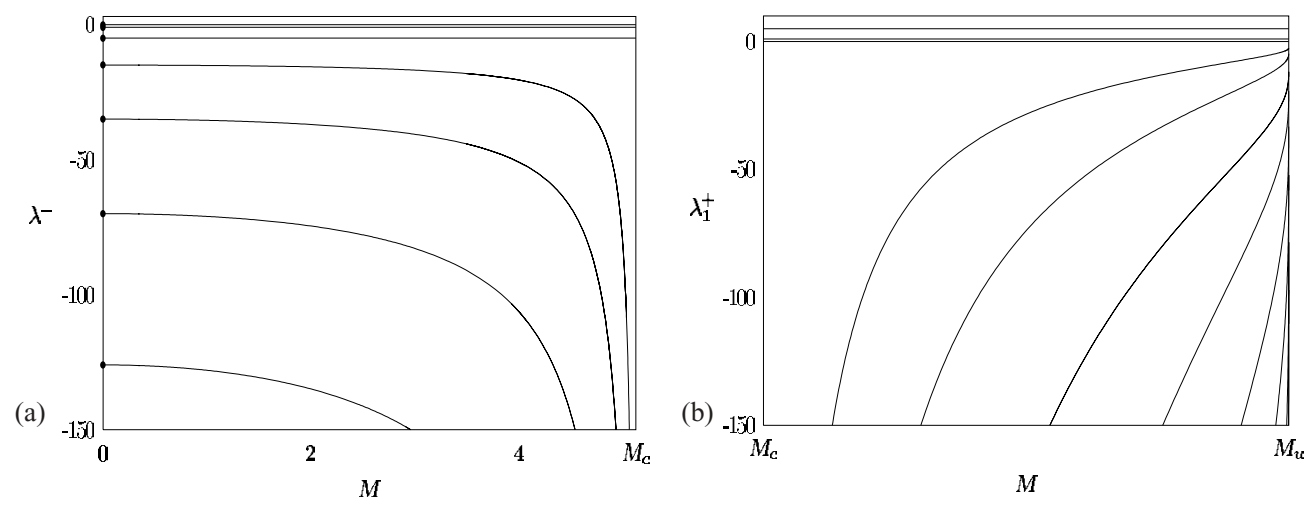

FIGURE 11. (a) Eigenvalues for the branch of spreading similarity solutions with solid dots for $M=0$ given by (5.15); (b) eigenvalues for the first branch of blow-up similarity solutions.

to note that $\bar{H}^{\prime \prime \prime \prime}(\bar{L})=-\sigma$ and therefore the linearized interface condition is given by

$$
\lambda \hat{L}=\hat{H}^{\prime \prime \prime}(\bar{L}) \text {. }
$$

It is convenient to eliminate $\hat{L}$ between (5.12) and (5.13) to yield the final form of the boundary conditions on $\hat{H}(\eta)$, [10]

$$
\hat{H}(\bar{L})=0, \quad \lambda \hat{H}^{\prime}(\bar{L})+\bar{H}^{\prime \prime}(\bar{L}) \hat{H}^{\prime \prime \prime}(\bar{L})=0,
$$

and similarly at the other interface, $\eta=-\bar{L}$. Therefore the linear stability problems for the three cases of droplet solutions (steady-state, spreading, and blow-up) are given by $(5.3,5.4)$ subject to $(5.14)$.

\subsection{Self-similar spreading droplet solutions, $\bar{H}^{-}(\eta)$}

First, we consider the stability of the spreading similarity solutions, $\sigma=-1$. In this case, the symmetry eigenvalues connected with spatial and temporal translations of the solution $(5.6,5.8)$ are negative, $\lambda_{T}=-5$ and $\lambda_{X}=-1$. The presence of these modes in the spectrum is an artefact of the use of similarity variables. As mentioned earlier, the definitions of the similarity variables, (3.3) and (3.5), depend on the parameters $x_{c}$ and $t_{c}$ describing the position and the critical (starting or ending) time of the similarity solution. The apparent stabilizing influence of the symmetry modes in this case is a consequence of the 'defocusing' nature of the spreading similarity solution. As $t \rightarrow \infty$, the solution becomes less sensitive to the values of $x_{c}$ and $t_{c}$ as it spreads out. This is analogous to the influence of the initial data on the long-time asymptotics for a solution of a diffusive problem. If appropriate values for $x_{c}$ and $t_{c}$ are obtained, then improved results can be found for the rate of convergence of $h(x, t)$ to the similarity solution $h \rightarrow \bar{H}^{-}(\eta) / \tau$; this is called the optimal similarity solution [64]. Otherwise, the rate of convergence to the similarity solution will be limited by $\lambda_{T}$ and $\lambda_{X}$.

Apart from the two symmetry eigenvalues, and the zero-mode $\lambda_{M}=0$ associated with existence of a continuous family of solutions, the rest of spectrum must be calculated numerically (see Figure 11a). Numerical calculation of the other eigenmodes follows 
from the discretization of (5.3) with appropriate boundary conditions, yielding a matrix eigenvalue problem that can be solved using the methods of numerical linear algebra $[63,65]$. For the limit $M \rightarrow 0$ we can make use of the fact that, to leading order, the spreading solution is given by the source-type similarity solution of the thin film equation, (4.20). To leading order, the linear stability problem for $\bar{H}^{-}(\eta)$ is also the same, and hence from [10], as $M \rightarrow 0$ the eigenvalues are given by

$$
\lambda^{-} \sim-\frac{k(k+1)(k+2)(k+3)}{24} \quad k=0,1,2,3, \cdots
$$

and the spectrum shows a continuous dependence on $M$ with $\lambda=O\left(\left(M_{c}-M\right)^{-1}\right)$ as $M \rightarrow M_{c}$ (except for the constants $\lambda_{T}=-5, \lambda_{M}=0$, and $\lambda_{X}=-1$ associated with the symmetry modes) - see Figure 11a. Since there are no positive eigenvalues, the entire branch of spreading similarity solutions (for all masses $M<M_{c}$ ) is linearly stable.

\subsection{Self-similar blow-up droplet solutions, $\bar{H}^{+}(\eta)$}

In contrast, for the blow-up solutions, $\sigma=1$, the symmetry eigenvalues are $\lambda_{T}=5$ and $\lambda_{X}=1$. These unstable modes are a consequence of the "focusing" nature of the blow-up solutions. If the appropriate values of $x_{c}$ and $t_{c}$ for the position and critical time for the self-similar blow-up singularity occurring in the solution are given, the solution will approach the optimal self-similar solution $h \rightarrow \bar{H}^{+}(\eta) / \tau$ as $t \rightarrow t_{c}$. However, if the solution $h(x, t)$ is blowing up, but either the position or the critical time has been incorrectly predicted, then the norm of the error $\left\|h(x, t)-\bar{H}^{+}(\eta) / \tau\right\|_{\infty}$ will diverge, hence the presence of instabilities might be concluded. Yet, by selecting the optimal similarity solution for the problem, this divergence can be suppressed. The optimal solution is the one specified by appropriate values of $x_{c}$ and $t_{c}$ such that there are no contributions from the symmetry modes [64] (i.e. $\epsilon=0$ in (5.6) and (5.8)). Consequently the presence of these positive eigenvalues due to symmetries of the PDE does not imply instability of the similarity solutions.

Again, apart from $\lambda_{X}, \lambda_{T}, \lambda_{M}$, the rest of the spectrum of $(5.3,5.14)$ must be calculated numerically. Again we observe that the spectrum is real and discrete for each $\bar{H}^{+}(\eta)$ solution, parametrized by its mass (see Figure 11b). For the first branch of blow-up solutions, $\bar{H}_{1}^{+}(\eta ; M)$, we note that apart from the symmetry modes, all of the other eigenvalues are negative, and hence we conclude that these solutions are stable. For the higher-order branches of multi-bump blow-up solutions, $\bar{H}_{n}^{+}(\eta)$ with $n=2,3, \cdots$ (see Figure 6), other positive eigenvalues are present, hence these multi-bump solutions are all unstable (see Figure 12). Therefore we conclude that the only stable self-similar route to finite-time blow-up is via one of the single-bump similarity solutions from the first branch (see Figure 8a). We briefly consider issues connected to the convergence to these solutions from more general initial data with $M>M_{c}$ in numerical simulations given in $\S 6.3,6.5$.

\subsection{Steady state droplet solutions, $\bar{H}^{0}(x)$}

We conclude with the stability analysis of the steady-state droplet solutions, (4.16). As in the case of the other compactly-supported solutions, the spatial translation symmetry of 


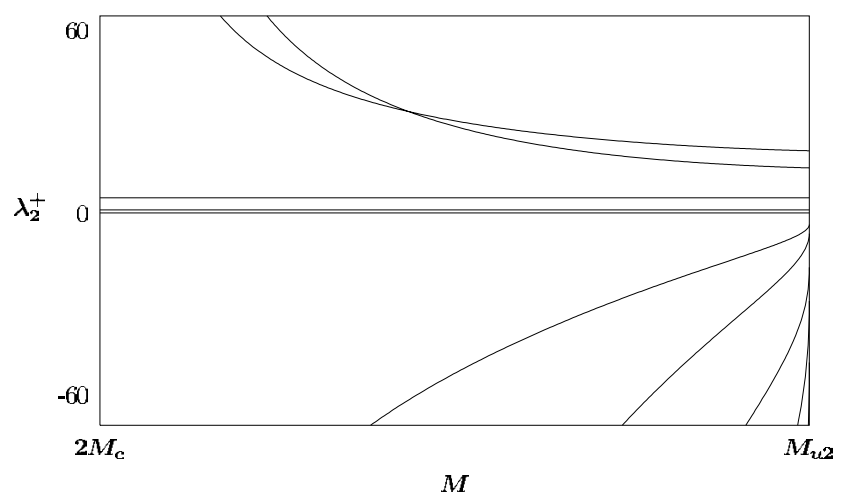

FIGURE 12 . Instability of the second branch of blow-up similarity solutions, $\bar{H}_{2}^{+}(\eta)$, indicated by the presence of two positive eigenvalues not associated with symmetries.

the PDE yields an eigenmode; for $\sigma=0,(5.8)$ yields a zero eigenvalue, $\lambda_{X}=0$. While the time-invariance of (3.4) for $\sigma=0$ is trivial (5.6) and does not contribute to the spectrum, another symmetry of the $\bar{H}^{0}(x)$ solutions takes its place. As described in Section 4.2, the $\bar{H}^{0}(x)$ are invariant under changes in the length of the interval of support (4.16). Hence letting $\bar{L} \rightarrow \bar{L}+\epsilon$ yields another steady-state droplet, and produces another zero eigenmode,

$$
\bar{H}^{0}(x) \sim \bar{H}^{0}(x)+\epsilon \hat{H}_{L}(x) e^{\lambda_{L} t}, \quad \hat{H}_{L}(x)=-\frac{1}{\bar{L}} \frac{d}{d x}\left(x \bar{H}^{0}(x)\right), \quad \lambda_{L}=0 .
$$

Note that for the case $\bar{L}=1$, where $\bar{H}^{0}(x)$ reduces to $h_{c}(x)$, this result matches the form of (5.6).

The influence of a perturbation of the mass (5.9) for these solutions also requires special attention. While steady-state droplets exist only for the critical mass, $M=M_{c}$, it is also true that for $\bar{L} \rightarrow 0$, these solutions are the continuous limits of self-similar spreading (blow-up) solutions with masses slightly less (more) than $M_{c}$, see (4.22), (4.25). Therefore, infinitesimal perturbations of the mass map steady-state droplets onto self-similar ones these solutions evolve, hence the influence of the perturbation grows with time. However, since the similarity solutions conserve mass, the eigenvalue must be $\lambda_{M}=0$. We conclude that perturbations of the mass of steady-state droplets must be described by a generalized eigenmode; analysis of this problem will involve a study of the center manifold of $\bar{H}^{0}(x)$ [38].

Numerically we find the spectrum for $\bar{H}^{0}(x)$ with $\bar{L}=1$ is

$$
\lambda^{0} \approx\{0,0,0,-785,-2045,-8592,-14803, \cdots\}
$$

with $\lambda_{k}^{0}=O\left(k^{4}\right)$ as $k \rightarrow \infty$. It is important to note that as mass approaches the critical mass, the limit of the eigenvalues of the steady states $\bar{h}(x)$ is not the set of eigenvalues of the compactly-support equilibrium, $\bar{H}^{0}(x)$, that is $\{\bar{\lambda}\} \not\left\{\lambda^{0}\right\}$ as $M \rightarrow M_{c}$. While it is true that in this limit, $\bar{h}(x) \rightarrow \bar{H}^{0}(x)$, the boundary conditions in the two cases are different, and this has a dramatic effect on stability; $\bar{h}(x)$ is unstable while $\bar{H}^{0}(x)$ is marginally stable. 


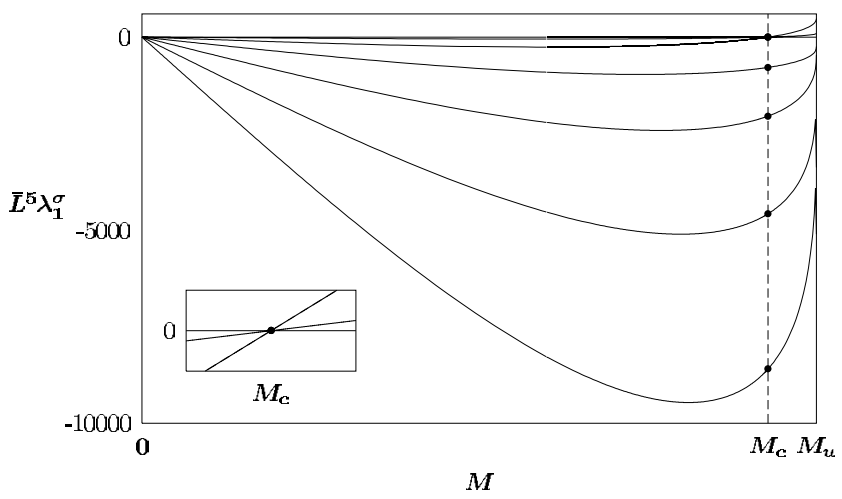

FiguRE 13. The continuity of the eigenvalues across the spreading, steady-state and blow-up droplet solutions. The inset detail shows the symmetry eigenvalues $\lambda_{X}, \lambda_{T}, \lambda_{M}$ near the critical mass $M_{c}$. The solid dots correspond to $\lambda^{0}(5.17)$.

For $\bar{H}^{0}(x)$ with $\bar{L} \neq 1$, the spectrum can be obtained from (5.17) by noting that the rescaling symmetry $(x, h, t) \rightarrow\left(x / \bar{L}, h / \bar{L}, t / \bar{L}^{5}\right)$ implies that $\lambda^{0} \rightarrow \lambda^{0} / \bar{L}^{5}$. A direct consequence is that the product $\bar{L}^{5} \lambda$ is scale-invariant for the steady-state droplets. For $M \neq M_{c}$ the similarity solutions are not invariant under changes in $\bar{L}$, but as $M \rightarrow M_{c}$ they do continuously approach $\bar{H}^{0}(x)$. We illustrate these facts in Figure 13, where it is shown that the product $\bar{L}^{5} \lambda^{\sigma}$ is continuous across the three classes of droplet solutions.

\section{Dynamics}

We conclude by presenting a series of numerical simulations of the PDE (1.1) with different forms of initial data. The simulations illustrate some of the predictions of the earlier sections, as well as exploring other issues that lie beyond the scope of the analysis. The simulations were carried out using implicit finite-difference methods specialized for thin film problems with singularities $[11,13,25,63]$.

\subsection{Dynamics starting from positive initial data}

While the family of nontrivial steady state solutions found in $\S 4.1$ were shown to be unstable in $\S 5.1$, they still play an important role in the dynamics of the PDE. These unstable equilibria are saddle points in the solution space for the problem; their stable and unstable manifolds partition the solutions of the PDE into basins of attraction for qualitatively different dynamics. This is illustrated in Figure 14. For initial data starting close to unstable steady states, infinitesimal perturbations can determine if the solution will: (a) blow-up in finite time with $h_{\max } \rightarrow \infty$ and $h_{\min } \rightarrow 0$ or (b) converge to the uniform steady state with $h_{\max }, h_{\min } \rightarrow M / 2$. The branch of uniform solutions is only stable for $M<2 \pi$, hence for larger masses, blow-up will be the generic dynamics for almost every initial condition. However, in $\S 6.4$ we show that even in these cases, the dynamics leading up to the eventual blow-up may not be trivial.

A more delicate problem is the analysis of dynamics of solutions with mass $M=n M_{c}$ for $n=1,2,3, \cdots$. In these cases, stable configurations of $n$ steady-state droplets $\bar{H}^{0}(x)$ exist. 


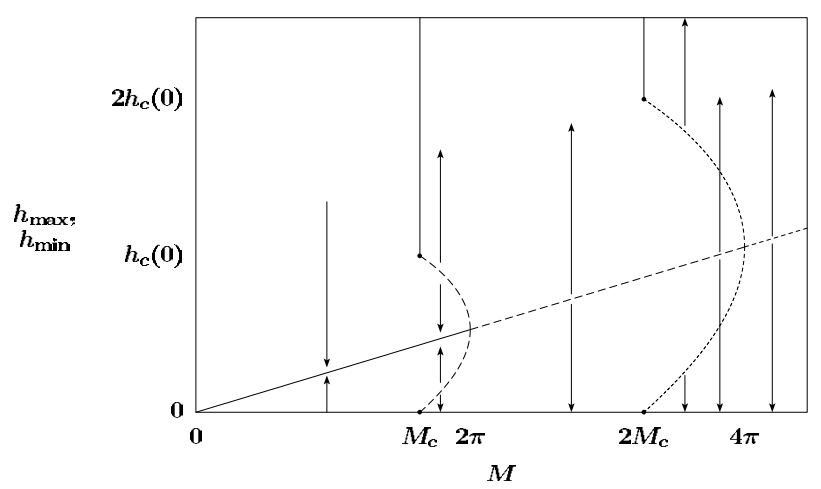

FIGURE 14. A schematic diagram for the basins of attraction for the steady-state solutions versus finite-time blow-up. The unstable steady-state solutions separate sets of solutions that lead to blow-up from those that converge to the uniform steady state $\bar{h}=M / 2$.

Numerical simulations of these problems remains an open question since the solutions are extremely sensitive to the mass, any perturbation of the mass will lead to blow-up as the end-product of a merging (coarsening $[14,35]$ ) instability, see $\S 6.4$. Issues related to the numerical solution of the PDE with compact initial data and the interaction of droplet solutions are discussed further below.

\subsection{Computing the dynamics of non-negative weak solutions}

As discussed above, different classes of compactly-supported solutions are central to the dynamics of (1.1). These are weak solutions since they have discontinuities in higher-order derivatives at their interfaces; the occurrence of such singular behavior makes computing such solutions very delicate. Extensive research has been done on the analysis of these solutions and the development of numerical schemes that can cope with their limited regularity $[16,17]$.

For our simulations, we make use of a regularization of the degeneracy in mobility coefficient, generalizing the analytical approach of Bernis \& Friedman [7] for the thin film equation, $h_{t}=-\left(f(h) h_{x x x}\right)_{x}$. Specifically, we numerically solve the modified PDE for $\varepsilon>0$,

$$
\frac{\partial h}{\partial t}=-\frac{\partial}{\partial x}\left(f_{\varepsilon}(h) \frac{\partial}{\partial x}\left[\frac{1}{3} h^{3}+\frac{\partial^{2} h}{\partial x^{2}}\right]\right)
$$

where $f_{\varepsilon}(h)$ is a regularized form of the mobility coefficient,

$$
f_{\varepsilon}(h)=\frac{h^{4}}{\varepsilon^{3}+h^{3}} .
$$

We have modified the notation from Bernis \& Friedman [7] so that $\varepsilon$ represents a thicknessscale for the thin film. For $h \gg \varepsilon, f_{\varepsilon}(h) \sim h$ and hence (1.3) is recovered from (6.1) for large initial data. This choice of regularization guarantees that positive initial data evolves to a 

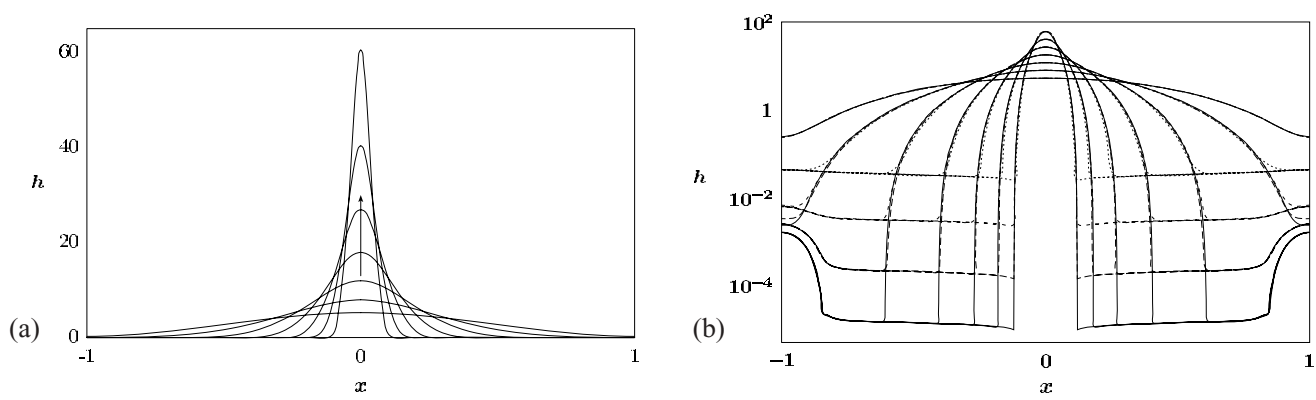

FigURE 15. (a) Numerical simulation of blow-up for a regularized weak solution of (6.1). (b) Timeprofiles from the same evolution shown on a log-scale to focus on the structure of the regularized interface for $\varepsilon=10^{-n}$ with $n=1,2,3,4$.

positive solution. Bertozzi \& Pugh $[16,17]$ show that as $\varepsilon \rightarrow 0$ the regularized solutions, when they exist, converge to a weak solution, of the original PDE, with zero contact angle (for almost every time). Thus we can use $(6.1,6.2)$ to approximate a weak solution; the fact that positivity holds for any fixed epsilon guarantees a numerical approximation that is well-behaved. In practice we find that the minimum follows $h_{\min }=O(\varepsilon)$.

To illustrate the influence of this regularization on the dynamics, we solved the periodic problem for (6.1) starting from positive initial data of the form $h_{0}(x)=\frac{1}{2} M+A \cos (\pi x)$ with $M>M_{c}$ and $A<M / 2$. As is expected, the solution approaches blows-up in finite time (see Figure 15a). However, before this solution blows-up, it appears to pinch-off to create a compactly supported mass involved in the blow-up. This is more evident in Figure $15 \mathrm{~b}$, where time-profiles of the solution computed with $\varepsilon=10^{-1}, 10^{-2}, 10^{-3}, 10^{-4}$ are plotted on a log-scale graph. For times while the solution remains numerically wellresolved, the minimum is bounded from below by $h_{\min }=O(\varepsilon)$. We also note that as $\varepsilon \rightarrow 0$, everywhere that the solution is finite, $h=O(1)$, it appears to converge, presumably to the weak solution of (1.1). Indeed, plotted normally, Figure 15a, there is no noticeable influence of the regularization on the dominant blow-up dynamics.

\subsection{Instability of the higher-order blow-up solutions}

One of the notable features of the set of generalized equilibria is the existence of the higherorder blow-up similarity solutions. In Figure 16 a we show a simulation starting from initial data given by a two-bump blow-up similarity with $\bar{L}=2$, i.e. $h(x, 0)=\bar{H}_{2}^{+}(x / 2) / 2$. It quickly destabilizes, as is expected from the presence of large positive eigenvalues for this solution (see Figure 12). By viewing the simulation in rescaled coordinates suggested by the similarity variables (3.3), we observe that the solution converges to a stable blow-up solution from the first branch $(n=1)$ as the the singularity is approached (see Figure 16b). It is interesting to note that while each of the two 'bumps' in the initial data have a structure that is closely related to the stable blow-up solution $\bar{H}_{1}^{+}(\eta)$, only one singularity, rather than two, ultimately occurs. An argument addressing this point and involving the form of the pressure function $p(x, t),(1.3)$, will be given in connection with the next simulation. 

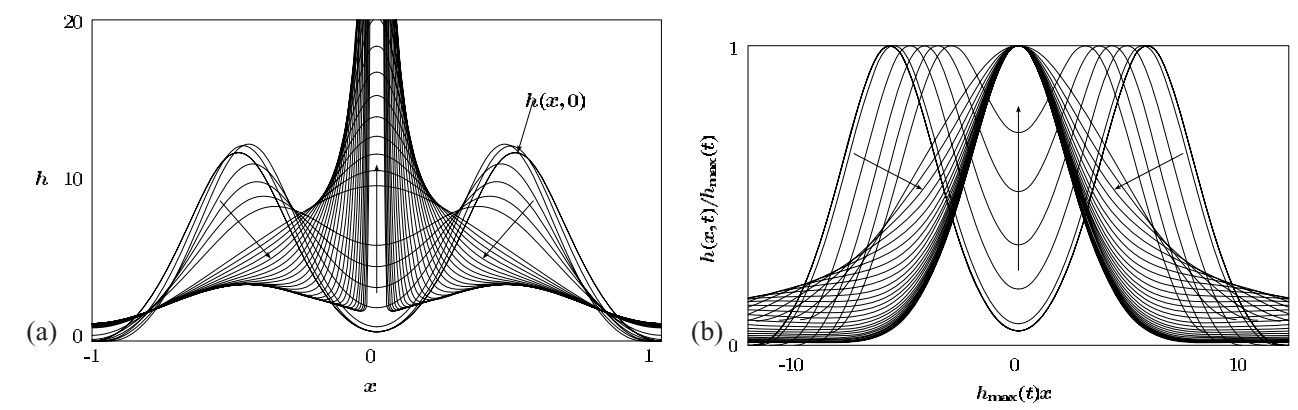

FIGURE 16. Instability of a multi-bump similarity solution: (a) time profiles for a solution approaching blow-up, (b) the same data in rescaled coordinates showing convergence to the stable similarity solution for blow-up.
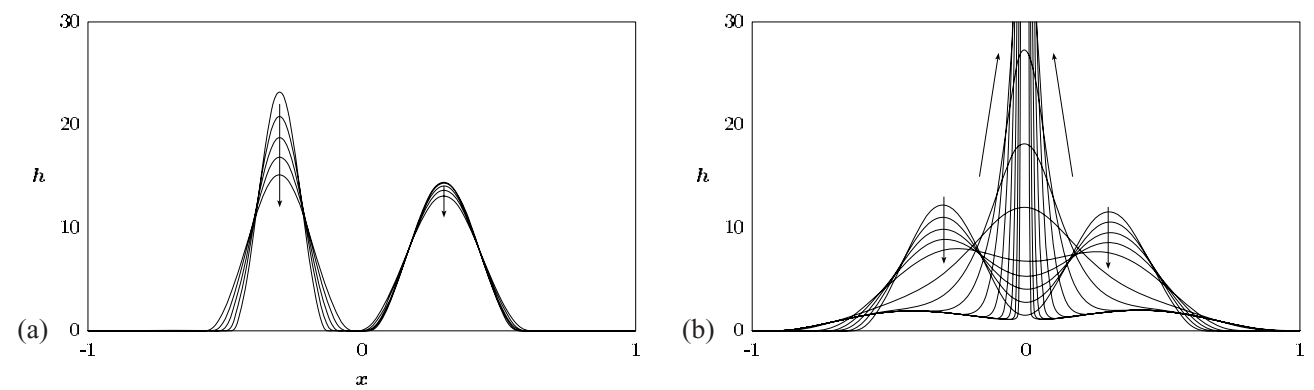

FIGURE 17. Evolution starting from two subcritical-mass droplets: (a) short-time self-similar spreading, (b) subsequent merging and eventual finite-time blow-up.

\subsection{Merging of subcritical solutions leading to blow-up}

An important property of degenerate diffusion equations like (1.1) is the nonlinear superposition principle for disjointly-supported weak solutions. That is, as long as their respective regions of support do not overlap, any combination non-negative weak solutions of (1.1) can be 'pasted together' in the domain to yield another solution, or droplet configuration [42,45]. For times when there is no overlap of domains, each droplet solution evolves independently of the others. An example of this is shown in Figure 17a, where two disjointly supported droplets, each with subcritical masses $M<M_{c}$, evolve according to the appropriate spreading-type similarity solutions, $\bar{H}^{-}(\eta)$. However, the initial data in this simulation was selected so that the total mass in the domain was supercritical, $M>M_{c}$, so finite-time blow-up can be expected to occur. How do we reconcile that such dissipative dynamics can lead to finite-time blow-up?

The resolution between these very different modes of dynamics lies in the transition (5.1e) that occurs when the two compactly-supported drops merge to become a 'single mass'. The dynamics of this simulation for longer times, after the drops have begun to merge, are shown in Figure 17b. If the total mass is supercritical, why should subcritical spreading behavior be expected for the initial regime of the dynamics? What is it that 

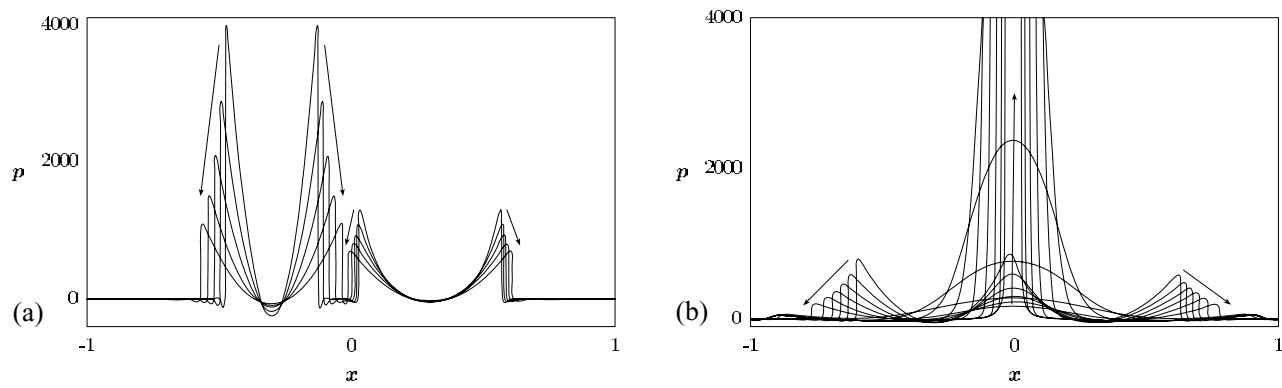

FIGURE 18. The pressure function $p=\frac{1}{3} h^{3}+h_{x x}$ corresponding to Figure 17: (a) decaying parabolic profiles with $p_{x x}>0$ for spreading solutions, (b) the formation of a growing pressure maximum at the position of the blow-up similarity solution.

determines the dynamics the solution follows? How is $\sigma$ in (3.4) selected? Some partial insights into the early stages of the merging process can be gained from a stability analysis of the spreading solutions with respect to non-compactly supported initial data [64]. However full descriptions of the transition in the dynamics from $\sigma=-1$ to $\sigma=1$ requires a more global view of the solution.

As a first step toward answering these questions, consider the form of the pressure function, $p=\frac{1}{3} h^{3}+h_{x x}$. The pressure for a spreading similarity solution $h=\bar{H}^{-}(\eta) / \tau$, is

$$
p(x, t)=\frac{\frac{1}{3} \bar{H}^{3}+\bar{H}^{\prime \prime}}{\tau^{3}}=\frac{1}{\tau^{3}}\left(\bar{P}+\frac{1}{2} \eta^{2}\right), \quad|\eta| \leqslant \bar{L}, \quad \tau \rightarrow \infty
$$

where the second equality is a consequence of equation (4.1). The pressure for a spreading similarity solution has a parabolic profile with $p_{x x}>0$ with decreasing amplitude and growing spatial support as time increases. The pressure is not continuous everywhere; there is a finite jump between the pressure at the interface $|\eta| \rightarrow \bar{L}^{-}$and the pressure outside the support, $p \equiv 0$ where $h \equiv 0$. When regularization is present, as in (6.1), jumps in the pressure will be somewhat smoothed (see Figure 18a). In contrast, the pressure for a blow-up similarity solution $h=\bar{H}^{+}(\eta) / \tau$, is given by

$$
p(x, t)=\frac{\frac{1}{3} \bar{H}^{3}+\bar{H}^{\prime \prime}}{\tau^{3}}=\frac{1}{\tau^{3}}\left(\bar{P}-\frac{1}{2} \eta^{2}\right), \quad|\eta| \leqslant \bar{L}, \quad \tau \rightarrow 0 .
$$

That is, the pressure has a local maximum, $p_{x x}<0$, at the blow-up position, $x_{c}$ (where $\eta=0$ ). Numerically, we observe that when the two pressure waves from the spreading solutions collide in the simulation (see the last time-profile in Figure 18a) they form a local maximum that evolves to produce blow-up (see Figure 18b). Note that the position of the maximum may shift during the evolution.

Returning to the simulation of the unstable multi-bump similarity solution in $\S 6.3$, we make use of the pressure to argue that while $h(x, t)$ initially had two maxima only one rather than two independent blow-up singularities should be expected. This is suggested by the fact that the corresponding $p(x, t)$ (still given by (6.4)) has only one local maximum that might determine the position of a blow-up singularity. 


\subsection{Weak vs. classical blow-up}

All of our simulations of blow-up share the same common dynamics: as the formation of the blow-up singularity is approached, the solution converges to a stable (single-bump) blow-up similarity solution, $\bar{H}^{+}(\eta)$. What is not the same in all of the simulations is that depending on the initial data, the nature of the convergence to $\bar{H}^{+}$can be different. We distinguish two cases:

(a) Weak Blow-up: at some time, pinch-off of the solution occurs somewhere in the domain, $h \rightarrow 0$, yielding a weak solution. This weak solution then evolves toward blow-up, $h\left(x_{c}, t\right) \rightarrow \infty$ as $t \rightarrow t_{c}$. For example, see Figure 15 .

(b) Classical Blow-up: pinch-off does not occur; the minimum of the solution $h(x, t)$ is bounded away from zero by a finite value (independent of the regularization). Convergence to the interface conditions for the compactly-supported similarity solutions (3.10) occurs by virtue of the limit $\tau \rightarrow 0$ as blow-up is approached. For example, see Figures $16 \mathrm{ab}$ and $17 \mathrm{~b}$.

In particular, to clarify case (b), suppose that $h_{\min }$ is constant, then in terms of the similarity variables, as blow-up is approached the minimum of the similarity solution is given by $\min _{\eta} H(\eta, s) \sim \tau h_{\min } \rightarrow 0$. Hence compact-support can be approached for the similarity solution even though $h(x, t)$ remains positive (see Figure 16). Which of these two cases occurs as blow-up is approached appears to be sensitive to the details of the form of the initial data.

Finally, we consider a simple way to study the convergence of the solution $h(x, t)$ to a self-similar solution as blow-up is approached. As described in Section 4, each blow-up similarity solution has a well-defined mass. However, in practice it is not clear how to accurately calculate the mass associated with the blow-up in the numerical solution. In both cases (a) and (b), it is difficult to clearly identify the interface position (in case (a) this is due to the need for regularization). However, we can determine the mass by relating it to a local property of the similarity solution - at the blow-up position $x_{c}$ we can calculate a local Bond number,

$$
B \equiv-\frac{h_{\max }^{3}}{\partial_{x x} h_{\max }},
$$

where the Bond number is classically defined as the ratio of the influence of body forces over the surface tension contributions to the pressure, see (1.3). This physically motivated quantity is scale-invariant ( $\tau$-independent) for the similarity solutions (3.3), $B=-\bar{H}^{3}(0) / \bar{H}^{\prime \prime}(0)$. A plot of the Bond number for the first branch of similarity solutions shows that it is a monotone increasing function of the mass (see Figure 19a). At the critical mass, $M_{c}$, the critical Bond number for the compact steady-states, $\bar{H}^{0}(x)$, is $B_{c}=4$. Spreading similarity solutions with $M<M_{c}$ have $B<4$, and the first branch of blow-up solutions cover the interval $4 \leqslant B \leqslant B_{u}$ where the upper value of the Bond number is $B_{u} \approx 4.27$ corresponding to the maximum-mass similarity solution, with $M=M_{u}$. Figure $19 \mathrm{~b}$ shows plots of $B(t)$ vs. $h_{\max }(t)$ for two simulations, one representing each of the two cases given above. For weak blow-up, case (b), we observe that after pinch-off occurs, $B$ rapidly converges to a constant value corresponding to some intermediate mass blow-up solution. For classical blow-up, case (a), convergence to a limiting value for $B$ 

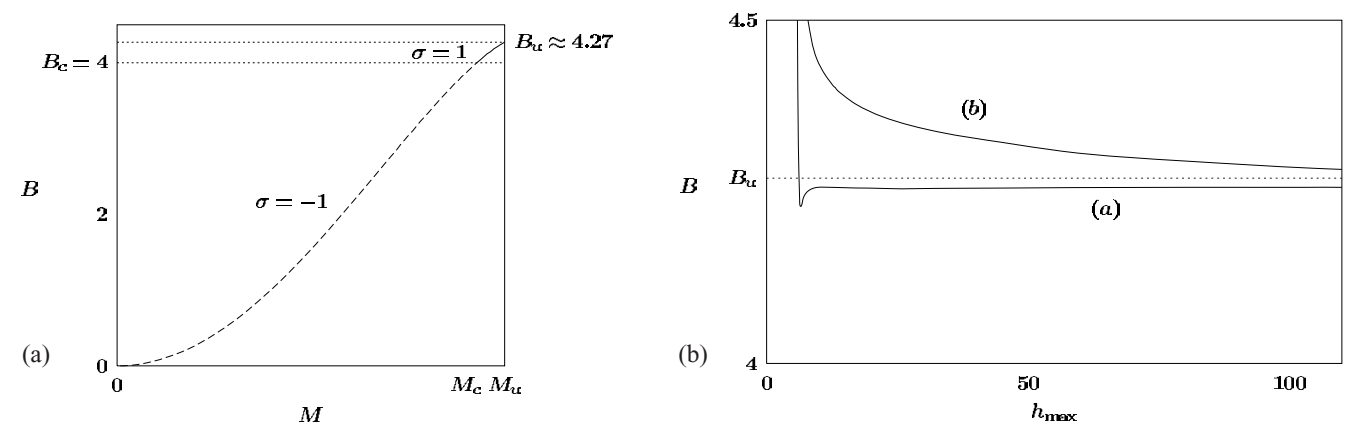

Figure 19. (a) The local Bond number (6.5) versus the mass for self-similar solutions, and (b) the evolution of the Bond number for examples of (a) weak blow-up, and (b) classical blow-up.

occurs much more slowly as mass flux out of the neighborhood of $x_{c}$ continues. Further work on the analysis of these cases is currently in progress.

\section{Conclusions}

This paper concerns equation (1.1) which is a critical case for the family of longwave unstable lubrication equations (1.2). We have identified a critical mass separating blow-up solutions from those that decay, and we have described the structure and stability of these solutions. Since we are considering a critical exponent, we find a richer set of solutions than might be expected for non-critical exponents $(m \neq n+2)$. That is, where other equations typically have locally unique similarity solutions, (1.1) has continuous families. Indeed the evolution within these continuous families leaves some open questions which are discussed in Sections 5 and 6.5. Our study benefited from the fact that the special case $n=1$ has some properties which make it more tractable for both rigorous and asymptotic analysis. Very recently, Slepčev \& Pugh [58] have examined the critical case $(m=n+2)$ of equation (1.2) for $0<n<3 / 2$. They have rigorously shown the existence of compactly supported self-similar solutions with single and multiple maxima, extending some of the results obtained here. This suggests that the dynamics of the critical case for $0<n<3 / 2$ may be analogous to the case $n=1$. This is a fertile area for future research. Moreover, the critical exponents for blow-up in higher dimensional problems for (1.2) is a challenging open question.

\section{Acknowledgements}

We would like to acknowledge helpful conversations with Elena Beretta on results in [5]. We also thank the anonymous referees for many helpful suggestions in the review process. This research was supported by NSF grants DMS-0074049, DMS-0244498, and DMS-0239125. ALB was supported in part by ONR grant N000140110290. TPW was supported in part by a fellowship from the Alfred P. Sloan foundation. AJB was supported in part by NSF grant DMS-9971969; he would also like to acknowledge the support of Duke University during several visits there. 


\section{References}

[1] Almgren, R., Bertozzi, A. L. \& Brenner, M. P. (1996) Stable and unstable singularities in the unforced Hele-Shaw cell. Phys. Fl. 8(6), 1356-1370.

[2] Bandle, C. \& Brunner, H. (1998) Blowup in diffusion equations: a survey. J. Comput. Appl. Math. 97(1-2), 3-22.

[3] Barenblatt, G. I. (1996) Scaling, Self-similarity, and Intermediate Asymptotics. Cambridge University Press.

[4] Barenblatt, G. I., Beretta, E. \& Bertsch, M. (1947) The problem of the spreading of a liquid film along a solid surface: A new mathematical formulation. Proc. Nat. Acad. Sci. 94, 10024-10030.

[5] Beretta, E. (1997) Selfsimilar source solutions of a fourth order degenerate parabolic equation. Nonlinear Anal. 29(7), 741-760.

[6] Beretta, E. (2001) Private communications.

[7] Bernis, F. \& Friedman, A. (1990) Higher order nonlinear degenerate parabolic equations. J. Diff. Eq. 83(1), 179-206.

[8] Bernoff, A. J. \& Bertozzi, A. L. (1995) Singularities in a modified Kuramoto-Sivashinsky equation describing interface motion for phase transition. Physica D, 85(3), 375-404.

[9] Bernoff, A. J., Bertozzi, A. L. \& Witelski, T. P. (1998) Axisymmetric surface diffusion: dynamics and stability of self-similar pinchoff. J. Statist. Phys. 93(3-4), 725-776.

[10] Bernoff, A. J. \& Witelski, T. P. (2002) Linear stability of source-type similarity solutions of the thin film equation. Appl. Math. Lett. 15(5), 599-606.

[11] Bertozzi, A. L. (1996) Symmetric singularity formation in lubrication-type equations for interface motion. SIAM J. Appl. Math. 56(3), 681-714.

[12] Bertozzi, A. L. (1998) The mathematics of moving contact lines in thin liquid films. Notices of the Am. Math. Soc. 45(6), 689-697.

[13] Bertozzi, A. L., Brenner, M. P., Dupont, T. F. \& Kadanoff, L. P. (1994) Singularities and similarities in interface flow. In: L. Sirovich (ed.) Trends and Perspectives in Applied Mathematics: Applied Mathematical Sciences 100, pp. 155-208. Springer-Verlag.

[14] Bertozzi, A. L., Grün, G. \& Witelski, T. P. (2001) Dewetting films: bifurcations and concentrations. Nonlinearity, 14(6), 1569-1592.

[15] Bertozzi, A. L. \& Pugh, M. (1996) The lubrication approximation for thin viscous films: regularity and long time behavior of weak solutions. Comm. Pur. Appl. Math. 49(2), 85-123.

[16] Bertozzi, A. L. \& Pugh, M. (1998) Long-wave instabilities and saturation in thin film equations. Comm. Pure Appl. Math. 51(6), 625-661.

[17] Bertozzi, A. L. \& Pugh, M. (2000) Finite-time blow-up of solutions of some long-wave unstable thin film equations. Indiana Univ. Math. J. 49(4), 1323-1366.

[18] Bertsch, M., Dal Passo, R., Garcke, H. \& Grün, G. (1998) The thin viscous flow equation in higher space dimensions. Adv. Diff. Eq. 3, 417-440.

[19] Brenner, M. P., Constantin, P., Kadanoff, L. P., Schenkel, A. \& Venkataramani, S. C. (1999) Diffusion, attraction and collapse. Nonlinearity, 12(4), 1071-1098.

[20] Bricmont, J. \& Kupiainen, A. (1994) Universality in blow-up for nonlinear heat equations. Nonlinearity, 7(2), 539-575.

[21] BudD, C. J. (2002) Asymptotics of multibump blow-up self-similar solutions of the nonlinear Schrödinger equation. SIAM J. Appl. Math. 62(3), 801-830.

[22] Budd, C. J. \& Galaktionov, V. (1998) Stability and spectra of blow-up in problems with quasi-linear gradient diffusivity. R. Soc. Lond. Proc. Ser. A Math. Phys. Eng. Sci. 454(1977), 2371-2407.

[23] Constantin, P., Dupont, T. F., Goldstein, R. E., Kadanoff, L. P., Shelley, M. J. \& Zhou, S.-M. (1993) Droplet breakup in a model of the Hele-Shaw cell. Physical Review E, 47(6), 4169-4181.

[24] Del Pino, M. \& Dolbeault, J. (2002) Best constants for Gagliardo-Nirenberg inequalities and applications to nonlinear diffusions. J. Math. Pures Appl. 81(9), 847-875. 
[25] Dupont, T. F., Goldstein, R. E., Kadanoff, L. P. \& Zhou, S.-M. (1993) Finite-time singularity formation in Hele Shaw systems. Phys. Rev. E, 47(6), 4182-4196.

[26] Ehrhard, P. (1994) The spreading of hanging drops. J. Colloid \& Interface Sci. 168(1), 242-246.

[27] Evans, S. C. (1998) Partial differential equations. American Mathematical Society.

[28] Fibich, G. (2001) Self-focusing in the damped nonlinear Schrödinger equation. SIAM J. Appl. Math. 61(5), 1680-1705.

[29] Filippas, S. \& Kohn, R. V. (1992) Refined asymptotics for the blowup of $u_{t}-\Delta u=u^{p}$. Comm. Pure Appl. Math. 45(7), 821-869.

[30] Galaktionov, V. A. \& Vázquez, J. L. (2002) The problem of blow-up in nonlinear parabolic equations. Discrete Contin. Dyn. Syst. 8(2), 399-433.

[31] Giga, Y. \& Kohn, R. V. (1985) Asymptotically self-similar blow-up of semilinear heat equations. Comm. Pure Appl. Math. 38(3), 297-319.

[32] Giga, Y. \& Kohn, R. V. (1987) Characterizing blowup using similarity variables. Indiana Univ. Math. J. 36(1), 1-40.

[33] Giga, Y. \& Kohn, R. V. (1989) Nondegeneracy of blowup for semilinear heat equations. Comm. Pure Appl. Math. 42(6), 845-884.

[34] Giga, Y. \& Kohn, R. V. (1989) Removability of blowup points for semilinear heat equations. In Differential Equations, pp. 257-264. Dekker.

[35] Glasner, K. B. \& Witelski, T. P. (2003) Coarsening dynamics of dewetting films. Phys. Rev. E, 67, 016302-1-12.

[36] Goldstein, R. E., Pesci, A. I. \& Shelley, M. J. (1993) Topology transitions and singularities in viscous flows. Phys. Rev. Lett. 70(20), 3043-3046.

[37] Grinfeld, M. \& Novick-Cohen, A. (1999) The viscous Cahn-Hilliard equation: Morse decomposition and structure of the global attractor. Trans. Am. Math. Soc. 351(6), 23752406.

[38] Guckenheimer, J. \& Holmes, P. (1990) Nonlinear Oscillations, Dynamical Systems, and Bifurcations of Vector Fields: Applied Mathematical Sciences 42. Springer-Verlag.

[39] Hocherman, T. \& Rosenau, P. (1993) On KS-type equations the evolution and rupture of a liquid interface. Physica $D, 67$.

[40] Holmes, M. H. (1995) Introduction to Perturbation Methods. Springer-Verlag.

[41] Kevorkian, J. \& Cole, J. D. (1996) Multiple Scale and Singular Perturbation Methods. SpringerVerlag.

[42] Laugesen, R. S. \& Pugh, M. C. (2000) Linear stability of steady states for thin film and Cahn-Hilliard type equations. Arch. Ration. Mech. Anal. 154(1), 3-51.

[43] Laugesen, R. S. \& Pugh, M. C. (2000) Properties of steady states for thin film equations. Euro. J. Appl. Math. 11(3), 293-351.

[44] Laugesen, R. S. \& Pugh, M. C. (2002) Energy levels of steady states for thin-film-type equations. J. Diff. Eq. 182(2), 377-415.

[45] Laugesen, R. S. \& Pugh, M. C. (2002) Heteroclinic orbits, mobility parameters and stability for thin film type equations. Electr. J. Diff. Eq. 95, 1-29.

[46] LeMesurier, B. J., Papanicolaou, G. C., Sulem, C. \& Sulem, P.-L. (1988) Local structure of the self-focusing singularity of the nonlinear Schrödinger equation. Phys. D, 32(2), 210-226.

[47] Levine, H. A. (1999) The role of critical exponents in blowup theorems. SIAM Rev. 32(2), 262-288.

[48] Merle, F. (1989) Limit of the solution of a nonlinear Schrödinger equation at blow-up time. J. Funct. Anal. 84(1), 201-214.

[49] Mitrinović, D. S., PeČArić, D. E. \& Fink, A. M. (1991) Inequalities Involving Functions and their Integrals and Derivatives. Kluwer Academic.

[50] Myers, T. G. (1998) Thin films with high surface tension. SIAM Rev. 40(3), 441-462.

[51] Newman, W. I. (1984) A Lyapunov functional for the evolution of solutions to the porous medium equation to self-similarity I. J. Math. Phys. 25(10), 3120-3123.

[52] Ockendon, J. R., Howison, S., Lacey, A. A. \& Movchan, A. (1999) Applied Partial Differential Equations. Oxford University Press, Oxford. 
[53] Oron, A., Davis, S. H. \& Bankoff, S. G. (1997) Long-scale evolution of thin liquid films. Rev. Mod. Phys. 69(3), 931-980.

[54] Dal Passo, R., Garcke \& GrÜN, H. G. (1998) On a fourth order degenerate diffusion equation: Global entropy estimates and qualitative behavior of solutions. SIAM J. Math. Anal. 29(2), 321-342.

[55] Plecháč, P. \& Šverák, V. (2001) On self-similar singular solutions of the complex GinzburgLandau equation. Comm. Pure Appl. Math. 54(10), 1215-1242.

[56] RotTsChäFER, V. \& KAPER, T. J. (2003) Geometric theory for multi-bump, self-similar, blowup solutions of the cubic nonlinear Schrödinger equation. Nonlinearity, 16(3), 929-961.

[57] Samarskit, A. A., Galaktionov, V. A., Kurdyumov, S. P. \& Mikhailov, A. P. (1995) Blow-up in quasilinear parabolic equations. Walter de Gruyter.

[58] SlepČEv, D. \& Pugh, M. C. (2003) Selfsimilar blowup of unstable thin-film equations. Preprint.

[59] Sz. NagY, B. v. (1941) Über Integralungleichungen zwischen einer Funktion und ihrer Ableitung. Acta Univ. Szeged. Sect. Sci. Math. 10, 64-74.

[60] Weinstein, W. I. (1982-83) Nonlinear Schrödinger equations and sharp interpolation estimates. Comm. Math. Phys. 87(4), 567-576.

[61] Whitham, G. B. (1974) Linear and Nonlinear Waves. Wiley-Interscience.

[62] Williams, M. B. \& Davis, S. H. (1982) Nonlinear theory of film rupture. J. Colloid \& Interface Sci. 90(1), 220-228.

[63] Witelski, T. P. (2002) Computing finite-time singularities in interfacial flows. Modern Methods in Scientific Computing and Applications, pp. 451-487. Kluwer.

[64] Witelski, T. P. \& Bernoff, A. J. (1998) Self-similar asymptotics for linear and nonlinear diffusion equations. Stud. Appl. Math. 100(2), 153-193.

[65] Witelski, T. P. \& Bernoff, A. J. (2000) Stability of self-similar solutions for van der Waals driven thin film rupture. Phys. Fluids, 11(9), 2443-2445.

[66] Witelski, T. P. \& Bernoff, A. J. (2000) Dynamics of three-dimensional thin film rupture. Phys. D, 147(1-2), 155-176.

[67] Zhang, W. W. \& Lister, J. R. (1999) Similarity solutions for van der Waals rupture of a thin film on a solid substrate. Phys. Fluids, 11(9), 2454-2462. 